\title{
The gas, metal, and dust evolution in low-metallicity local and high-redshift galaxies ${ }^{\star}$
}

\author{
A. Nanni ${ }^{1}$, D. Burgarella ${ }^{1}$, P. Theulé ${ }^{1}$, B. Côté ${ }^{2,3}$, and H. Hirashita ${ }^{4}$ \\ 1 Aix Marseille Univ, CNRS, CNES, LAM, Marseille, France \\ e-mail: ambra.nanni@lam.fr \\ 2 Konkoly Observatory, Research Centre for Astronomy and Earth Sciences, MTA Centre for Excellence, \\ Konkoly Thege Miklos 15-17, 1121 Budapest, Hungary \\ 3 ELTE Eötvös Loránd University, Institute of Physics, Budapest, Hungary \\ ${ }^{4}$ Institute of Astronomy and Astrophysics, Academia Sinica, Astronomy-Mathematics Building, AS/NTU, No. 1, Sec. 4, \\ Roosevelt Road, Taipei 10617, Taiwan
}

Received 27 February 2020 / Accepted 18 June 2020

\begin{abstract}
Context. The chemical enrichment in the interstellar medium (ISM) of galaxies is regulated by several physical processes: star birth and death, grain formation and destruction, and galactic inflows and outflows. Understanding such processes and their relative importance is essential to following galaxy evolution and the chemical enrichment through the cosmic epochs, and to interpreting current and future observations. Despite the importance of such topics, the contribution of different stellar sources to the chemical enrichment of galaxies, for example massive stars exploding as Type II supernovae ( $\mathrm{SNe}$ ) and low-mass stars, as well as the mechanisms driving the evolution of dust grains, such as for example grain growth in the ISM and destruction by SN shocks, remain controversial from both observational and theoretical viewpoints.

Aims. In this work, we revise the current description of metal and dust evolution in the ISM of local low-metallicity dwarf galaxies and develop a new description of Lyman-break galaxies (LBGs) which are considered to be their high-redshift counterparts in terms of star formation, stellar mass, and metallicity. Our goal is to reproduce the observed properties of such galaxies, in particular (i) the peak in dust mass over total stellar mass (sMdust) observed within a few hundred million years; and (ii) the decrease in sMdust at a later time.

Methods. We fitted spectral energy distribution of dwarf galaxies and LBGs with the "Code Investigating GALaxies Emission", through which the total stellar mass, dust mass, and star formation rate are estimated. For some of the dwarf galaxies considered, the metal and gas content are available from the literature. We computed different prescriptions for metal and dust evolution in these systems (e.g. different initial mass functions for stars, dust condensation fractions, SN destruction, dust accretion in the ISM, and inflow and outflow efficiency), and we fitted the properties of the observed galaxies through the predictions of the models.

Results. Only some combinations of models are able to reproduce the observed trend and simultaneously fit the observed properties of the galaxies considered. In particular, we show that (i) a top-heavy initial mass function that favours the formation of massive stars and a dust condensation fraction for Type II SNe of around 50\% or more help to reproduce the peak of sMdust observed after $\approx 100 \mathrm{Myr}$ from the beginning of the baryon cycle for both dwarf galaxies and LBGs; (ii) galactic outflows play a crucial role in reproducing the observed decline in sMdust with age and are more efficient than grain destruction from Type II SNe both in local galaxies and at high-redshift; (iii) a star formation efficiency (mass of gas converted into stars) of a few percent is required to explain the observed metallicity of local dwarf galaxies; and (iv) dust growth in the ISM is not necessary in order to reproduce the values of sMdust derived for the galaxies under study, and, if present, the effect of this process would be erased by galactic outflows.
\end{abstract}

Key words. galaxies: evolution - galaxies: dwarf - galaxies: high-redshift - galaxies: ISM

\section{Introduction}

Understanding the metals and dust cycle in the interstellar medium (ISM) of galaxies is essential to study their properties and evolution, and to interpret different observations of local and very distant galaxies. Indeed, dust shapes the spectra of galaxies by absorbing the stellar radiation in the ultraviolet (UV) and visible wavelengths and re-emitting it in the infrared (IR) bands, while the metals in the gas phase are responsible for various emission and absorption lines. Different works have been devoted to the study of the dust con-

* Full Table 3 is only available at the CDS via anonymous ftp to cdsarc.u-strasbg. fr (130.79.128.5) or via http://cdsarc. u-strasbg.fr/viz-bin/cat/J/A+A/641/A168 tent in galaxies in the Local Universe (Lisenfeld \& Ferrara 1998; Dwek 1998; Hirashita 1999; da Cunha et al. 2010; Zhukovska 2014; Schneider et al. 2016; Rémy-Ruyer et al. 2015; Popping et al. 2017; Gioannini et al. 2017; De Vis et al. 2017, 2019; McKinnon et al. 2018; Ginolfi et al. 2018) as well as in a variety of high-redshift galaxies (Graziani et al. 2020; Burgarella et al. 2020), including quasars at $z>6$ (Valiante et al. 2014; Calura et al. 2014; Mancini et al. 2015; Aoyama et al. 2017; Wang et al. 2017) and sub-millimetre galaxies (Rowlands et al. 2014; Cai et al. 2020).

Dust is condensed in Type II supernovae (SNe II) remnants and in the dense outflows of low-mass stars at the end of their evolution, during the thermally pulsing asymptotic giant branch (TP-AGB) phase. Massive stars evolve on short 
timescales $(<30 \mathrm{Myr})$, while AGB stars need a longer time to begin enriching the ISM. Furthermore, massive and low-mass stars produce different types of elements and dust. Massive stars are factories of oxygen and silicon (Limongi \& Chieffi 2018; Marassi et al. 2019), while low-mass stars can release large amounts of carbon already at very low metallicities (Nanni et al. 2013, 2014). Originating from exploding white dwarfs in binary systems, SN Ia enrich the ISM predominantly with iron on a timescale comparable with the evolution of low-mass stars that, contrary to massive stars, end their evolution as white dwarfs. The role of $\mathrm{SNe}$ as dust producers is still debated. On the one hand, it has been argued that shock waves propagating after their explosion heat up the gas and can severely reduce the amount of condensed dust (Nozawa et al. 2011; Bocchio et al. 2016; Bianchi \& Schneider 2007). On the other hand, for $\mathrm{SNe}$ II, Gall et al. (2014) found evidence for the presence of $0.1-$ $0.5 M_{\odot}$ of large grains in SN2010j1 and thus able to survive the passage of the reverse shock. In addition, recent observations from Matsuura et al. (2019) of SN1987 show that dust might re-condense in the cooling gas that experienced the passage of the forward shock. In the former case, this might indicate that dust can be efficiently reformed after being destroyed by shocks. The presence of dust has also been suggested for Type Ia SNe (Gomez et al. 2012). Around TP-AGB stars, dust can be condensed in considerable amounts (Ventura et al. 2012; Nanni et al. 2013, 2014), but as these objects evolve on longer timescales, their contribution may be irrelevant at early epochs.

Several different investigations have shown that dust growth in the ISM of galaxies helps to reproduce the large mass of observed dust that may be difficult to explain from stellar sources only (Asano et al. 2013; Zhukovska 2014; Michałowski 2015; Mancini et al. 2015; Leśniewska \& Michałowski 2019). However, dust accretion in the ISM may be difficult to explain from the microphysical point of view (Ferrara et al. 2016). Icy mantles can contribute to depleting elements from the gas phase, but they are expected to evaporate in the presence of a strong radiation field (Ferrara et al. 2016). Furthermore, the probability that two silicon-bearing molecules can encounter in the grain icy mantle is very low (Ceccarelli et al. 2018). Some laboratory experiments show that dust could accrete in low-temperature environments by the addition of molecules on the surface of dust grains (Rouillé et al. 2015). This process might be prevented in space by the formation of icy mantles on the grain surface (Ceccarelli et al. 2018), but grains may also grow when the icy mantle evaporates (Rouillé et al. 2020). Theoretical studies and experiments also suggest charged grains and molecules could favour the process of grain growth (Hollenstein 2000; Bleecker et al. 2006). Such a possibility has been investigated in the conditions typical of the Milky Way (Zhukovska et al. 2016) but further studies are needed. Grain growth in the ISM also seems to be related to the properties of galaxies. In particular, it appears to be more efficient in galaxies with greater stellar mass, gas density, and metallicity (Mancini et al. 2015; Schneider et al. 2016; Popping et al. 2017; Graziani et al. 2020), or enhanced if the ISM is turbulent (Mattsson et al. 2019; Mattsson 2020). Because of these controversial results, it is important to critically examine dust growth in comparison with observational data.

From the Atacama Large Millimiter Array (ALMA) detection, in Burgarella et al. (2020) we proposed a template for the dust emission of Lyman-break galaxies (LBGs) at the end of reionisation, which might be the first dust grains in the Universe. Along with rest-frame UV and optical data, this template is used to fit and model the multi-wavelength emission of these objects. From the derived physical parameters, we build a chemi- cal evolutionary model that explains the submillimetre (submm) detection (or non-detection) of high-redshift LBGs at the end of reionisation without any dust growth in the ISM. This model successfully explains different diagnostic diagrams (e.g. the evolution of the dust content in these galaxies). The non-detection is explained by dust destruction from shocks produced by $\mathrm{SNe}$ plus removal in the circum- and intergalactic media by outflows.

In this paper, we interpret the observations of low-metallicity dwarf galaxies (Rémy-Ruyer et al. 2015) in the Local Universe from the Dwarf Galaxy Survey (DGS, Madden et al. 2013) together with the LBGs, which share similar properties in terms of star formation activity, metallicity, and stellar mass. The spectral energy distribution (SED) of these galaxies is fitted as discussed in Burgarella et al. (2020). In particular, we aim to simultaneously reproduce the available constraints for such galaxies: total dust mass, star formation rate (SFR), metallicity, dust-to-gas ratio, gas fraction, age, and, for a few local galaxies, circumgalactic dust. By doing this, we identify the critical processes that drive the evolution of baryons in these galaxies: star formation efficiency, metal and dust enrichment from stellar sources (SNe II, TP-AGB stars, and SNe Ia), dust destruction from $\mathrm{SNe}$, galactic outflows, and the role of dust accretion in the ISM.

The paper is organised as follows. In Sect. 2 we describe the two samples of galaxies. In Sect. 3 we explain the prescriptions adopted for following the gas and dust evolution in the ISM of galaxies. In Sect. 4 we present the method for fitting the SED of galaxies and for comparing the model predictions with the properties derived. In Sect. 5 the results are provided, while Sect. 6 is devoted to discussing the results of this work in comparison with results from the literature. Our conclusions are provided in Sect. 7.

\section{Selected sample}

In this work, we analyse two data samples of DGS galaxies and LBGs for which the SED fitting was performed in Burgarella et al. (2020) using the Code Investigating GALaxies Emission (CIGALE), (Boquien et al. 2019). In CIGALE, the synthetic spectra are calculated from the energy balance between the photons absorbed and re-emitted by dust. Emission and absorption lines of gas are also included. The physical properties of the galaxies are estimated through the analysis of the likelihood distribution. The DGS galaxies form a low-metallicity $(12+\log (\mathrm{O} / \mathrm{H})<8.5)$ subsample of the galaxies presented in Rémy-Ruyer et al. (2015) for which the photometric data from the infrared to the submm range are available. The SED is then built by adding the photometry at shorter wavelengths (UV, B and R) taken from the NASA Extragalactic Database (NED). We use the most recent data corresponding to the integrated photometry of the galaxy. Employing data at short wavelengths allows us to better constrain the stellar mass and the SFR of the selected galaxies. Lyman-break galaxies were selected in the rest-frame UV and observed by ALMA. Burgarella et al. (2020) used the detections to build an IR SED template which is employed to re-fit all the objects individually. The SED fitting was performed employing a Chabrier initial mass function (IMF) in CIGALE with stellar mass in the range $0.1 \leq M_{*} \leq 100 M_{\odot}$ (Bruzual \& Charlot 2003). A top-heavy IMF is not available in the current version of CIGALE and will be a future improvement in the code. For the galaxies selected, CIGALE provides a good fit between the synthetic and observed photometry (reduced $\chi^{2} \leq 5$ ) for 31 out of 48 DGS galaxies and for 18 LBGs, which represents the two samples studied here. 


\section{Model for chemical evolution of galaxies}

In order to model the chemical evolution of the ISM of galaxies, we need to understand how the gas, metals, and dust evolve with time given the IMF of the stars, the star formation history (SFH) of the galaxy, and the theoretical yields from the stars.

\subsection{Basic assumptions and parameters}

The stars in the galaxies considered are assumed to form with a certain SFR. Consistently with the SED fitting performed in CIGALE, the functional form of the SFR used through this work is the "delayed" one:

$\mathrm{SFR}=C_{\mathrm{SFR}} \frac{t}{\tau^{2}} e^{-t / \tau}$

where the SFR is given in $M_{\odot} \mathrm{yr}^{-1}, \tau$ is a characteristic timescale, and $C_{\mathrm{SFR}}$ is a constant defined in such a way that the total mass of stars formed in the galaxy is normalised to one solar mass:

$C_{\mathrm{SFR}}=\frac{1 M_{\odot}}{\int_{0}^{t_{\mathrm{end}}} \operatorname{SFR}\left(t^{\prime}\right) \mathrm{d} t^{\prime}-M_{\mathrm{ev}}}$,

where $M_{\mathrm{ev}}$ is the total mass released into the ISM from the evolving stars and $t_{\text {end }}=13$ Gyr.

In this work we tested two different types of IMF for the stars: the Chabrier IMF (Chabrier 2003) and a "top-heavy" IMF defined as:

$\mathrm{IMF}=C_{\mathrm{IMF}} m^{-\alpha}$,

with $\alpha=1,1.35,1.5$. The constant $C_{\mathrm{IMF}}$ is computed in such a way that the integral of the IMF is normalised to one solar mass:

$C_{\mathrm{SFR}}=\frac{1 M_{\odot}}{\int_{M_{\min }}^{M_{\max }} \operatorname{IMF}\left(m^{\prime}\right) \mathrm{d} m^{\prime}}$,

where $M_{\min }$ and $M_{\max }$ are the minimum and maximum masses within which the theoretical metal yields are computed. Different metal yields available in the literature (characterised by diverse values of $M_{\min }$ and $M_{\max }$ ) are tested in this work (see Sect. 5.2.6). A top-heavy IMF implies a higher number of SNe II: the lower the value of $\alpha$, the larger the number of SNe II.

\subsection{Gas and metals}

Given the gas and metal yields for stars, the IMF, SFR, and outflows, we are able to describe the evolution of the mass of gas and chemical element, $i$, in the ISM. Under the aforementioned assumptions, the evolution of the mass of gas and metals is:

$$
\begin{aligned}
\frac{\mathrm{d} M_{\mathrm{g}}}{\mathrm{d} t}= & \int_{M_{\mathrm{L}}}^{M_{\mathrm{U}}} \operatorname{IMF} \times \operatorname{SFR}\left(t-t^{\prime}\right) f_{\mathrm{g}}\left[M_{*}\left(t^{\prime}\right), Z\left(t-t^{\prime}\right)\right] \mathrm{d} M- \\
& -\operatorname{SFR}(t)-\operatorname{ML} \times \operatorname{SFR}(t)+M L \frac{I}{O} \times \operatorname{SFR}(t),
\end{aligned}
$$

$$
\begin{aligned}
\frac{\mathrm{d} M_{\mathrm{i}}}{\mathrm{d} t}= & \int_{M_{\mathrm{L}}}^{M_{\mathrm{U}}} \operatorname{IMF} \times \operatorname{SFR}\left(t-t^{\prime}\right) f_{\mathrm{i}, \mathrm{g}}\left[M_{*}, Z\left(t-t^{\prime}\right)\right] \mathrm{d} M- \\
& -\operatorname{SFR}(t) f_{\mathrm{i}, \mathrm{g}}(t)-\operatorname{ML} \times \operatorname{SFR}(t) f_{\mathrm{i}, \mathrm{g}}(t),
\end{aligned}
$$

where the first term is the integral between the minimum $\left(M_{\mathrm{L}}\right)$ and the maximum $\left(M_{\mathrm{U}}\right)$ mass of stars that have a life-time $t^{\prime}$ and are enriching the ISM at time $t$ with a mass gas fraction, $f_{\mathrm{g}}$, or metal fraction, $f_{\mathrm{i}, \mathrm{g}}$. The stars enriching the ISM at a certain time were formed at $t-t^{\prime}$ and have metallicity $Z\left(t-t^{\prime}\right)$. The fractions of gas and metal ejected into the ISM depend on the metallicity and on the stellar mass $M_{*}$. The contribution to the ISM enrichment is weighed for the IMF. The second term of the equations is the astration of gas and metals due to the formation of stars. The chemical enrichment of the ISM and astration due to star formation (given by the first and second terms of Eqs. (5) and (6)) are computed by means of the OMEGA code (Côté et al. 2017; Ritter et al. 2018, One-zone Model for the Evolution of GAlaxies). Starting from these calculations, the overall evolution of gas and metals is followed by an external routine. At each time-step the galactic outflow is assumed to be proportional to the SFR through the "mass-loading factor" (ML), which assumes the outflow to be due to the stellar feedback in the ISM (Murray et al. 2005). A corresponding mass of gas and metals is removed from the ISM. The code allows the possibility to introduce a galactic inflow of pristine gas provided by the last term of Eq. (5). Such a term is proportional to ML and is regulated by the ratio between the mass of inflowing and outflowing gas $(\mathrm{I} / \mathrm{O})$. The total initial mass of baryons in the galaxy, $M_{\mathrm{bar}}$, is another input quantity of our model. This quantity is entirely composed of gas at the beginning of the simulation, and it is partially converted into stars according to Eq. (1). We set $M_{\text {bar }}$ to a certain mass of gas, $M_{\text {gas }}$, which is a multiple of the stellar mass, $M_{\text {stars }}$ normalised to $1 M_{\odot}$ at $13 \mathrm{Gyr}$, and we add to $M_{\mathrm{gas}}$ the mass of gas ejected by all the stars during their evolution, $M_{\mathrm{ev}}$ in Eq. (2). The value of $M_{\mathrm{ev}}$ is evaluated with the OMEGA code.

\subsection{Dust}

We assume that the dust formed in supernovae remnants, around evolved low-mass stars, and in the ISM is composed predominantly of silicates (olivine and pyroxene), amorphous carbon dust (characterised by different fractions of $\mathrm{sp}^{2}$ and $\mathrm{sp}^{3}$ bonds), and metallic iron. Carbon is also partially locked in hydrocarbons, such as in polycyclic aromatic hydrocarbons. Iron dust can be partially included in silicates or be in different chemical forms (e.g. iron oxides, iron carbides, iron sulphides, and pyrrhotite). Throughout this work, we consider iron-free silicates (olivine and pyroxene), amorphous carbon dust, and metallic iron. We are interested in estimating the total mass of dust rather than its specific chemical composition. To this purpose the possible presence of dust species of different chemical composition from the specific one considered here, such as iron-rich silicates or other dust species including carbon or iron atoms, will not significantly alter the predicted mass of dust with respect to our estimates. Starting from the calculations from OMEGA, we then follow the evolution of the dust content in the ISM, by assuming at each time step that a fraction of the available metals is condensed into dust grains. In addition, we take into account different physical processes that change the mass of dust as a function of time: outflows, dust destruction from SN shock waves that propagate in the ISM, and dust growth in the ISM. Consistently with the calculations of gas and metal evolution, we assume that the inflow material is composed of pristine gas that does not change the total content of dust. For each dust species, the variation of the dust content in the galaxy is given by:

$\frac{\mathrm{d} M_{\mathrm{d}, \mathrm{i}}}{\mathrm{d} t}=f_{\mathrm{i}, \mathrm{d}} \frac{\mathrm{d} M_{\mathrm{i}, \mathrm{d}}}{\mathrm{d} t}-\mathrm{ML} \times \operatorname{SFR}(t) \delta_{\mathrm{i}, \mathrm{d}}(t)-\frac{\mathrm{d} M_{\mathrm{d}, \mathrm{i}, \mathrm{destr}}}{\mathrm{d} t}+\frac{\mathrm{d} M_{\mathrm{d}, \mathrm{i}, \mathrm{gr}}}{\mathrm{d} t}$,

where the first term of the equation takes into account the metal enrichment from the composite stellar population and the astration computed with OMEGA and $f_{\mathrm{i}, \mathrm{d}}$ is the condensation fraction 
of dust ${ }^{1}$. Different condensation fractions for $\mathrm{SNe}$ and TP-AGB stars are assumed. The quantity $\mathrm{d} M_{\mathrm{i}, \mathrm{d}} / \mathrm{d} t$ in the first term is the maximum mass of each dust species that can be formed and is computed by dividing $\mathrm{d} M_{\mathrm{i}} / \mathrm{d} t$ in Eq. (6) by the mass of each element and by multiplying for the mass of the nominal monomer of dust given its stoichiometric formula. A fraction of dust is lost due to outflows and is provided by the second term on the right-hand side of Eq. (7), where $\delta_{\mathrm{i}, \mathrm{d}}(t)$ is the dust-to-gas ratio at time " $\mathrm{t}$ ". The term $\mathrm{d} M_{\mathrm{d}, \mathrm{i}, \mathrm{destr}} / \mathrm{d} t$ is the variation of the mass of dust due to the dust destruction from $\mathrm{SN}$ shocks and $\mathrm{d} M_{\mathrm{d}, \mathrm{i}, \mathrm{gr}} / \mathrm{d} t$ is dust growth in the ISM. We discuss the different mechanisms separately in the following sections.

\subsubsection{Dust condensation fractions for TP-AGB stars and $\mathrm{SNe}$}

For TP-AGB stars, the chemistry of dust is mainly determined by the number of carbon atoms in the stellar atmosphere over the number of oxygen ones ( $\mathrm{C} / \mathrm{O}$ ratio). For $\mathrm{C} / \mathrm{O}<1$, all the carbon atoms are locked in the very stable carbon monoxide molecules (CO) and the remaining oxygen is present in the atmosphere to form molecules and dust: the star is classified as oxygen-rich. For $\mathrm{C} / \mathrm{O}>1$, an excess of carbon is present in the atmosphere from which molecules and dust are formed: the star is classified as carbon-rich. Normally, the value of $\mathrm{C} / \mathrm{O}$ is less than one, but during the TP-AGB evolution, values of $\mathrm{C} / \mathrm{O}>1$ are attained as a consequence of third dredge-up events that follow the thermal pulse (Herwig 2005). Around oxygen-rich TP-AGB stars, a certain fraction of silicon, oxygen, and magnesium are used to build silicate dust (mainly olivine and pyroxene) and, in smaller amounts, metal oxides, while during their carbon-rich phase, the main dust species produced is carbon dust. Other species such as silicon carbide and metallic iron are also produced but in smaller amounts (Höfner \& Olofsson 2018). The chemistry of the dust formed around $\mathrm{SNe}$ II is instead more uncertain, since it depends on the mixing between the $\mathrm{C}$ and $\mathrm{O}$ layers which determines the amount of such species locked into CO molecules (Nozawa et al. 2003).

Here, we consider two different sets of condensation fractions for the different species formed around TP-AGB stars or in $\mathrm{SN}$ remnants. Around low-mass stars, the condensation fraction of silicate and carbon dust can reach up to 50-60\% (or slightly more) during the superwind phase when the star loses most of its mass (Ventura et al. 2012; Nanni et al. 2013, 2014), while for SN remnants the dust-to-metal mass fraction is estimated to be between $30 \%$ and $60 \%$ without considering the effect of dust destruction from the reverse shock and/or possible dust reformation (Marassi et al. 2019).

Following Nanni et al. (2013), for each time-step we assume the following condensation fractions for the different species for TP-AGB stars: $f_{\mathrm{py}}=0.3$ for pyroxene, $f_{\mathrm{ol}}=0.3$ for olivine, $f_{\text {car }}=0.5$ for carbon dust, and $f_{\text {ir }}=0.01$ for metallic iron. Different condensation fractions for the dust species formed in $\mathrm{SNe}$ II are considered. Given the uncertainties in the dust condensation fraction for SNe II, we tested a range of values between 0.1 and close to 1 for all dust species. In cases where the condensation fraction is close to the maximum, we set the condensation fraction of carbon to 0.5 , because some should be available in the atmosphere to form CO molecules (Spyromilio et al. 2001).

\footnotetext{
1 We define the condensation fraction of a dust species as the ratio between the number of atoms condensed into the dust grain to the total that can condense for the least abundant among the atomic species that forms a certain dust species.
}

Despite being a simplified approach, as dust yields are not consistently computed in the circumstellar envelopes of TP-AGB stars and in SN remnants for any of the metal yields considered in this work, this strategy allows us to test the results for different choices of the metal yields available in the literature.

\subsubsection{Dust destruction from SN shock waves}

We adopt a macroscopic description for dust destruction due to the passage of SN forward shocks in the ISM. We assume that all the dust species are destroyed with the same efficiency by such a process. The timescale that regulates the destruction from $\mathrm{SN}$ shock waves is given by:

$\tau_{\mathrm{d}}=\frac{M_{\mathrm{g}}(t)}{\epsilon R_{\mathrm{SN}}(t) M_{\mathrm{swept}}}$,

where $M_{\mathrm{g}}$ is the gas mass in the ISM which evolves according to Eq. (5). The quantity $M_{\text {swept }}$, given in $M_{\odot}$, is a model parameter that provides the amount of the ISM mass that is swept up by each SN event, and $\epsilon$ is the destruction efficiency. The quantity $R_{\mathrm{SN}}$ is instead the $\mathrm{SN}$ rate, which depends on the $\mathrm{SFH}$ and the IMF. For each dust species $i$, the destruction term is therefore given by:

$$
\frac{\mathrm{d} M_{\mathrm{d}, \mathrm{i}, \mathrm{destr}}}{\mathrm{d} t}=\frac{M_{\mathrm{d}, \mathrm{i}}}{\tau_{\mathrm{d}}} .
$$

In this work we consider: $M_{\text {swept }}=1000,6800 M_{\odot}$, while $\epsilon=0.1$ in all cases (McKee et al. 1989). $M_{\text {swept }}=6800 M_{\odot}$ represents the standard case in the literature (e.g. Dwek et al. 2007), while the assumption $M_{\text {swept }}=1000 M_{\odot}$ has also been considered on the basis of the calculations performed by Nozawa et al. (2006).

\subsubsection{Dust growth in the ISM}

Here, we analyse the efficiency of the dust growth process in the standard framework where molecules are added from the gas phase to the grain, forming the bulk of silicates (olivine or pyroxene), amorphous carbon, or metallic iron. No icy mantle formation is considered in this work. Different parameters are adopted in order to model such a process. Due to the lack of detailed laboratory measurements of grain growth at low temperatures, many of these parameters are uncertain. We treat such a process following grain growth and the variation of the size of each dust species as a function of time, as calculated in Nanni et al. (2013, 2014). We assume accretion to start on pre-existing grains (seed nuclei) on which other molecules from the gas phase are added to form the bulk of the grain. Such grains can be reprocessed in the ISM by SN shocks or aggregate in dense clouds modifying their size distribution. For each of the dust species, the overall reaction for the formation of the grain needs to be chosen a priori. The reactions assumed in this work are provided in Table 1. Carbon dust is formed from acetylenic radicals $\left(\mathrm{C}_{2} \mathrm{H}_{(0<=y<=4)}\right)$ on the grain surface. Here, we only consider the formation of carbon through the addition of $\mathrm{C}_{2} \mathrm{H}_{2}$ because we are interested in estimating the total amount of dust formed in the ISM. The exact pathway of grain formation is uncertain and such an investigation is beyond the scope of this paper. The possible destruction of dust due to the sputtering of the grains by $\mathrm{H}_{2}$ molecules is neglected in this work because this process is efficient above $\approx 1000$ K (Gail \& Sedlmayr 1999; Nanni et al. 2013). The variation of the grain size is regulated by the equation:

$\frac{\mathrm{d} a_{\mathrm{i}}}{\mathrm{d} t}=V_{0, \mathrm{i}} J_{\mathrm{i}}^{\mathrm{gr}}$ 
Table 1. Overall reactions adopted for modelling dust accretion in the ISM.

\begin{tabular}{lcc}
\hline \hline Species & Reactions & Molecules \\
\hline Olivine & $2 \mathrm{Mg}+\mathrm{SiO}+3 \mathrm{H}_{2} \mathrm{O} \rightarrow \mathrm{Mg}_{2} \mathrm{SiO}_{4}(s)+3 \mathrm{H}_{2}$ & $\mathrm{Mg}, \mathrm{SiO}, \mathrm{H}_{2} \mathrm{O}$ \\
Pyroxene & $\mathrm{Mg}+\mathrm{SiO}+2 \mathrm{H}_{2} \mathrm{O} \rightarrow \mathrm{MgSiO}_{3}(\mathrm{~s})+2 \mathrm{H}_{2}$ & $\mathrm{Mg}, \mathrm{SiO}, \mathrm{H}_{2} \mathrm{O}$ \\
$\mathrm{Fe}(\mathrm{s})$ & $\mathrm{Fe} \rightarrow \mathrm{Fe}(\mathrm{s})$ & $\mathrm{Fe}$ \\
$\mathrm{C}(\mathrm{s})$ & $\mathrm{C}_{2} \mathrm{H}_{2} \rightarrow 2 \mathrm{C}(\mathrm{s})+\mathrm{H}_{2}$ & $\mathrm{C}_{2} \mathrm{H}_{2}$ \\
\hline
\end{tabular}

Notes. The molecules and atoms in the gas phase from which each dust species is formed are also listed.

where we assume the initial size of dust grains to be $0.01 \mu \mathrm{m}$ (Hirashita \& Kuo 2011) which is derived from the size distribution of grains in our Galaxy (Mathis et al. 1977). If smaller grains are initially available in the ISM dust growth in the ISM may occur more rapidly (Hirashita 2012). The quantity $V_{0, \mathrm{i}}$ is the volume of the nominal monomer of each dust species and the growth rate, $J_{i}^{\mathrm{gr}}$, is instead evaluated as:

$J_{\mathrm{i}}^{\mathrm{gr}}=\min \left(\frac{s_{\mathrm{i}}}{s_{\mathrm{j}}} \alpha_{\mathrm{i}} n_{\mathrm{j}} v_{\mathrm{th}, \mathrm{j}}\right)$,

where $s_{\mathrm{j}}$ and $s_{\mathrm{i}}$ are the stoichiometric coefficient in the reaction for the species in the gas phase and for the monomer of dust, respectively. The quantity $\alpha_{\mathrm{i}}$ is a unitless parameter known as the "sticking coefficient" and represents the probability that a species in the gas phase sticks to the grain surface. The value of $\alpha_{\mathrm{i}}$ varies from 0 to 1 . On the basis of recent experiments at low temperature, the reaction between $\mathrm{SiO}$ molecules and the grain surface is expected to have zero energy barrier, which corresponds to a $\alpha_{\mathrm{i}}$ of 1 (Rouillé et al. 2015). Here, we assume $\alpha_{\mathrm{i}}=1$ for all the dust species. However, such an assumption represents an upper limit of the efficiency of grain accretion. The quantity $n_{\mathrm{j}}$ is the number density of the species $j$ in the gas phase involved in the formation of the dust grain, computed as:

$n_{\mathrm{j}}=\epsilon_{\mathrm{j}} n_{\mathrm{H}}$

where $\epsilon_{\mathrm{j}}$ is the abundance of the element $j$ and we adopt $n_{\mathrm{H}}=$ $10^{3} \mathrm{~cm}^{-3}$ (Hirashita 2000, 2012). For larger values of the gas density, grain growth would proceed more rapidly. At each timestep, the quantity $\epsilon_{\mathrm{j}}$ in the gas phase is computed by subtracting the fraction of each element condensed into dust grains from the total available. The incorporation of $\mathrm{CO}$ molecules into grains is not considered. Indeed, carbon monoxide is a very stable molecule that does not contribute to the accretion of dust grains. The fraction of carbon monoxide in the gas phase is estimated to be around $40 \%$ of the total amount of carbon (Lodders 2010; Agúndez \& Wakelam 2013). The abundance of $\mathrm{SiO}, \mathrm{Mg}, \mathrm{H}_{2} \mathrm{O}$, $\mathrm{C}_{2} \mathrm{H}_{2}$, and $\mathrm{Fe}$ in the gas phase is therefore estimated as the abundance of $\mathrm{Si}, \mathrm{Mg}, \mathrm{O}, \mathrm{C}$, and $\mathrm{Fe}$, respectively, neither locked in dust grains nor in $\mathrm{CO}$ molecules. The quantity $v_{\mathrm{th}, \mathrm{j}}$, is its thermal velocity, given by:

$v_{\mathrm{th}, \mathrm{j}}=\sqrt{\frac{k_{\mathrm{B}} T_{\mathrm{gas}}}{2 \pi m_{\mathrm{j}}}}$,

where $k_{\mathrm{B}}$ is the Boltzmann constant, $T_{\text {gas }}=25 \mathrm{~K}$ is the gas temperature, and $m_{\mathrm{j}}$ is the mass of the gas species. From the grain size it is possible to directly compute the total amount of dust produced in the dense phase of the ISM:

$\frac{\mathrm{d} M_{\text {growth,i }}}{\mathrm{d} t}=\frac{4 \pi}{3} \frac{\mathrm{d} a_{\mathrm{i}}}{\mathrm{d} t} a_{\mathrm{i}}^{2} \rho_{\mathrm{i}} n_{\mathrm{s}, \mathrm{i}}$ where $\rho_{\mathrm{i}}$ is the mass density of the dust species $i$, and $n_{\mathrm{s}, \mathrm{i}}$ is the number of seed nuclei. We estimate the total number of seed particles $n_{\mathrm{s}, \mathrm{i}}$ by dividing the mass of each dust species by the mass of a dust grain which depends on its size, as done in Asano et al. (2013). With this choice we implicitly assume that all the dust grains already present in galaxies can act as seed particles on which other molecules can accrete. The number of seed nuclei is expected to change as the ISM is progressively enriched by newly formed dust grains of a certain typical size and will depend on the different physical processes that modify the number of grains (e.g. shattering, coagulation). The larger the number of seed nuclei, the faster the dust growth in the ISM. If star formation mainly occurs at the beginning of the cycle (which is the case for the galaxies considered here), a further increase of the mass of dust in the ISM is mainly due to grain growth in the ISM. In this regime, the number of seed nuclei can be assumed to be roughly constant (if new seeds are not formed in the ISM) and equal to the total mass of a certain dust species divided by the current mass of the grain for each dust species, $i$ as computed in Asano et al. (2013).

\section{Method}

The observed photometry for galaxies is fitted with the code CIGALE. The features of the code are explained in Boquien et al. (2019) while the assumptions adopted for computing the synthetic spectra are discussed in Burgarella et al. (2020). The SED fitting procedure of the sources described in Sect. 2 allows us to derive the best value for the parameter $\tau$ in Eq. (1) for each of the galaxies, as well as the total mass of stars and dust, and the SFR. Furthermore, throughout this paper we use the specific mass of dust (sMdust), i.e. the mass of dust divided by the mass of stars, specific SFR (sSFR), i.e. the SFR divided by the mass of stars, and age. For DGS galaxies, other properties are available from the literature: metallicity, gas fraction, dust-to-gas ratio, and circumgalactic dust fraction.

The properties derived are compared with the output of a set of chemical evolutionary tracks obtained by varying the input parameters described in Sect. 3. The different combinations of the parameters adopted in Sect. 3 (IMF, condensation fraction, stellar mass range of the SN progenitors, efficiency of the galactic outflow, SN destruction, and initial gas content in the ISM) are listed in Table 2. For all the models (one at each time-step) inside the different chemical evolution tracks, we find the residual between the properties of the galaxies predicted by the chemical evolution calculation and the ones derived from the SED fitting of CIGALE:

$R_{\mathrm{gal}}^{2}=\sum_{\mathrm{k}}^{N_{\mathrm{k}}} \frac{\left(f_{\mathrm{obs}, \mathrm{k}}-f_{\mathrm{th}, \mathrm{k}}\right)^{2}}{\sigma_{\mathrm{k}}^{2}}$,

where $k$ represents the property of the galaxy. For the error on the age we adopted the maximum value between the uncertainty 
Table 2. Parameters adopted in the simulations of metal and dust evolution described in Sect. 3.

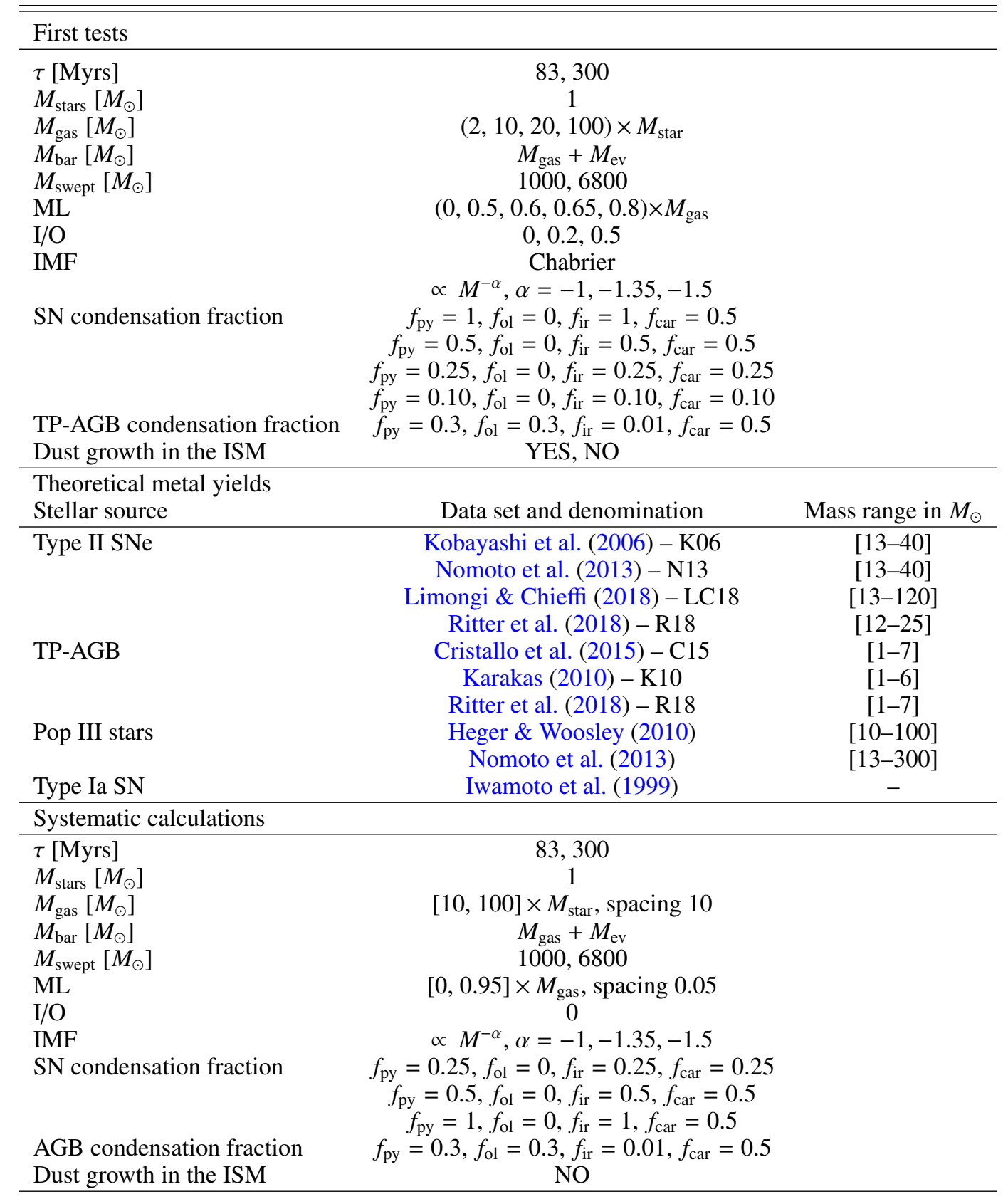

Notes. First tests are run in order to select the reference parameters adopted to run systematic calculations. The stellar mass produced after 13 Gyr is always normalised to $1 M_{\odot}$. Different theoretical metal yields are tested in Sect. 5.2.6.

given by CIGALE and $10 \%$ of the age. The metallicity and the gas mass are taken from Rémy-Ruyer et al. (2013, 2014) for DGS galaxies. We compute the total mass of gas by selecting from Rémy-Ruyer et al. (2014) the atomic and molecular hydrogen mass. The total hydrogen gas is then multiplied by the mean atomic weight of the galaxy $\left(\mu_{\mathrm{gal}}\right)$. The molecular hydrogen mass is derived from a metallicity-dependent conversion factor between the $\mathrm{CO}$ and $\mathrm{H}_{2}$ masses $\left(M_{\mathrm{H} 2, \mathrm{z}}\right)$. All the data adopted are provided in Table A.1. In case only upper limits are available, we adopt the maximum possible value. The error associated to the gas fraction and to the dust-to-gas is assumed to be $100 \%$ given the uncertainties affecting the conversion between $\mathrm{CO}$ and $\mathrm{H}_{2}$ abundances. For LBGs, we do not have direct measurements of the metallicity and of the gas content, and therefore we do not use such quantities for fitting the galaxies. The estimates of the circumgalactic dust fraction are taken from McCormick et al. (2018) for three of the DGS galaxies in the sample that are He210, NGC 1569, and NGC 5253.

From the residual $R_{\text {gal }}$ we calculate the probability density distribution (in analogy with the $\chi^{2}$ distribution), $p=$ $\exp \left(-R_{\text {gal }}^{2} / 2\right)$. Each of the properties of the galaxies is then estimated through the likelihood analysis by computing its average value and standard deviation by using the probability density distribution among all the calculated models as weights. The predicted values in different diagnostic diagrams (e.g. sMdust vs. sSFR) are compared with the ones derived from the SED fitting with CIGALE or taken from the literature. This procedure allows us to constrain the properties of individual galaxies. 
A. Nanni et al.: The gas, metal, and dust evolution in low-metallicity local and high-redshift galaxies

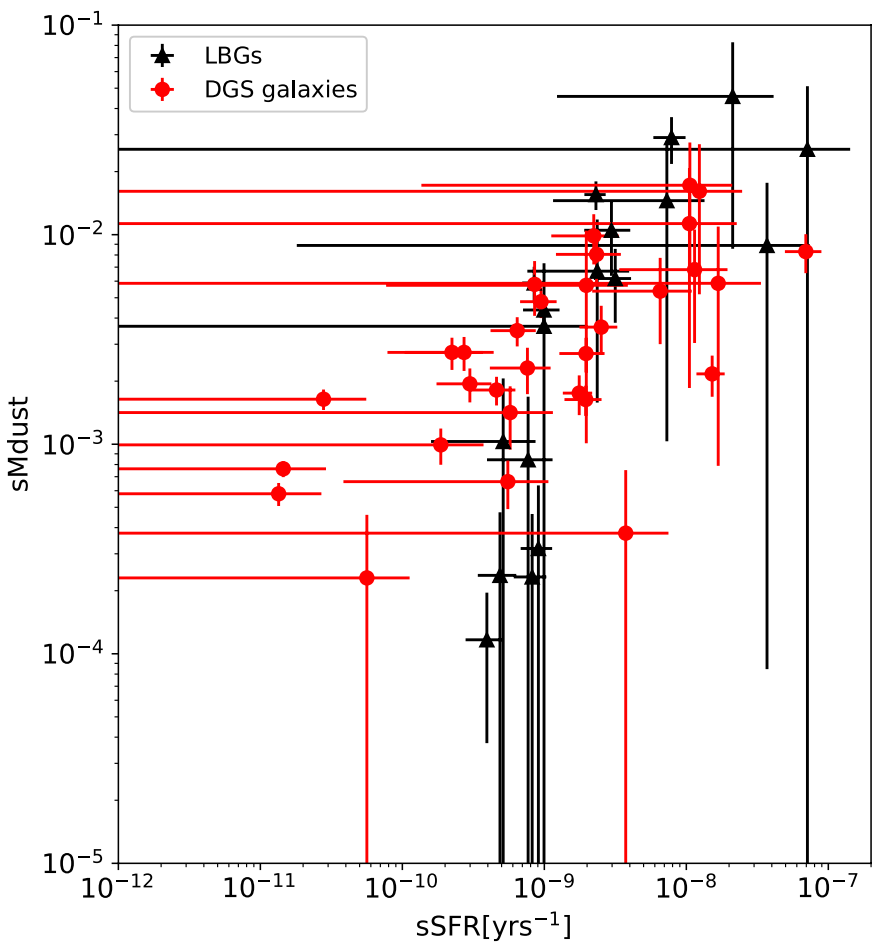

Fig. 1. sMdust against sSFR derived from the best fit performed with the code CIGALE for LBGs (black triangles) and DGS galaxies (red dots). See also Burgarella et al. (2020) for all the details.

\section{Results}

\subsection{Properties of galaxies derived from the SED fitting}

Here, we discuss the main properties derived for the galaxies considered from the SED fitting procedure. The SED fitting performed for the DGS galaxies in this work in which the IR data from Rémy-Ruyer et al. (2015) are combined with UV and optical photometry (see Sect. 2) allow us to better constrain the stellar mass and SFR of these galaxies with respect to the ones in Rémy-Ruyer et al. (2015) where only IR data were employed.

The average value of $\tau$ is estimated to be of 83 Myr for LBGs, while for DGS galaxies, we selected two representative values to run the chemical evolutionary models which are of $\tau=83$ Myr for those objects with $\tau<100$ Myr (5 galaxies), and the average value of $\tau=300 \mathrm{Myr}$ for the others 26 galaxies. Lyman-break galaxies are characterised by stellar population no older than $\approx 700 \mathrm{Myr}$, while stellar populations up to $\approx 800 \mathrm{Myr}$ are derived for DGS. As shown in Fig. 1, the SSFR of DGS galaxies is between $\approx 10^{-11}$ and $\approx 10^{-7} \mathrm{yrs}^{-1}$, while for LBGs the range is narrower, between $\approx 10^{-9}$ and $\approx 10^{-7} \mathrm{yrs}^{-1}$. The sMdust is between $\approx 10^{-4}$ and $\approx 10^{-2}$ for DGS galaxies, while LBGs attain larger values up to $\approx 6 \times 10^{-2}$. In the plot shown in Fig. 1 , the evolutionary time increases from right to left. This represents a relevant diagnostic diagram largely employed throughout this work and in the literature. The main features for both the DGS galaxies and LBGs in the sMdust against sSFR (and age) shown in Fig. 1, are (i) a peak at the beginning of the cycle and (ii) a later decrease. Lyman-break galaxies attain a value of sMdust larger than DGS galaxies, while the decline of sMdust is faster. The large error bars in of sMdust obtained for some of the galaxies are due to the non detection for these objects of the dust continuum emission for which only upper limits are available.

For DGS galaxies, we have additional information on the metallicity and on the gas content (see Rémy-Ruyer et al. 2013, 2014 , and references therein). The metallicity values are $7.1 \lesssim$
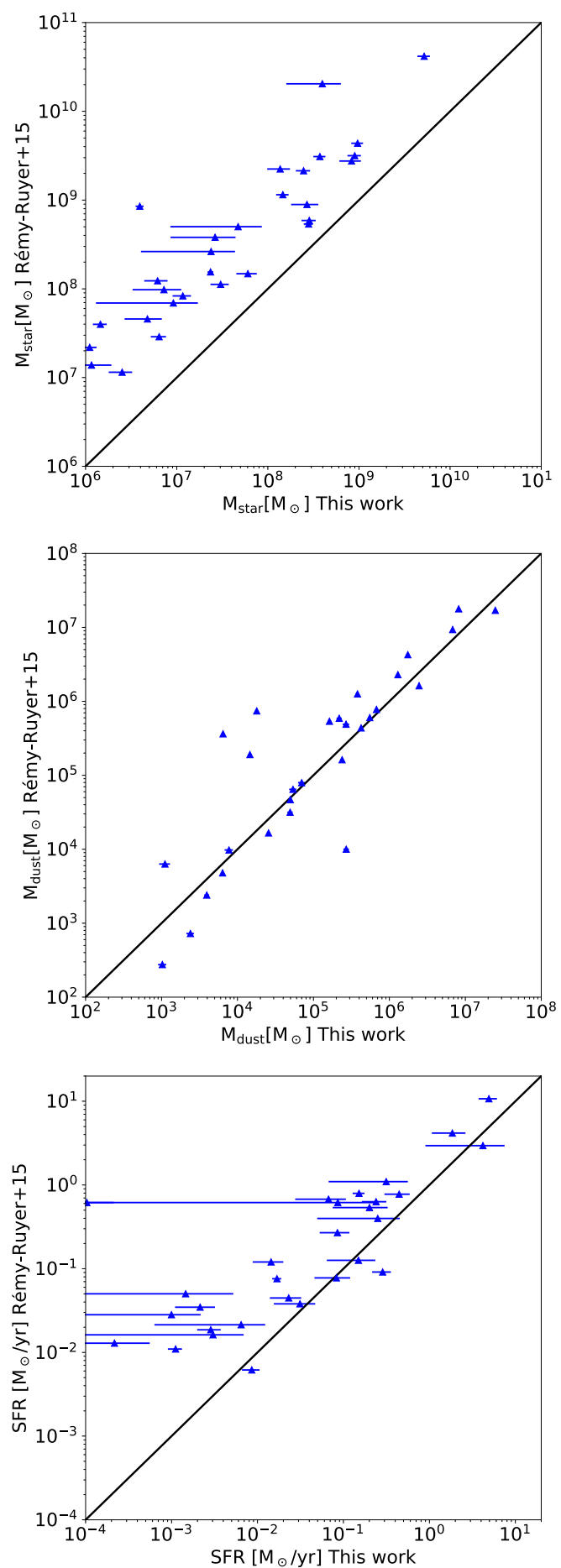

Fig. 2. Upper panel: stellar mass derived by Rémy-Ruyer et al. (2015) vs. that derived in Burgarella et al. (2020). Middle and lower panels: same as in the upper panel but for the mass of dust and SFR, respectively.

$12+\log (\mathrm{O} / \mathrm{H}) \lesssim 8.4$, the gas fraction is $0.4 \lesssim f_{\text {gas }} \lesssim 1$, and the dust-to-gas ratio is $10^{-5} \lesssim \mathrm{D} / \mathrm{G} \lesssim 10^{-3}$, where this latter quantity has been estimated by considering the dust mass derived in the analysis presented here. All the properties of DGS galaxies derived from the SED fitting performed with CIGALE are provided in Table A.1 together with all plots showing the best-fitting spectra (Fig. A.1). For LBGs properties and SED fitting we refer to the work of Burgarella et al. (2020). 


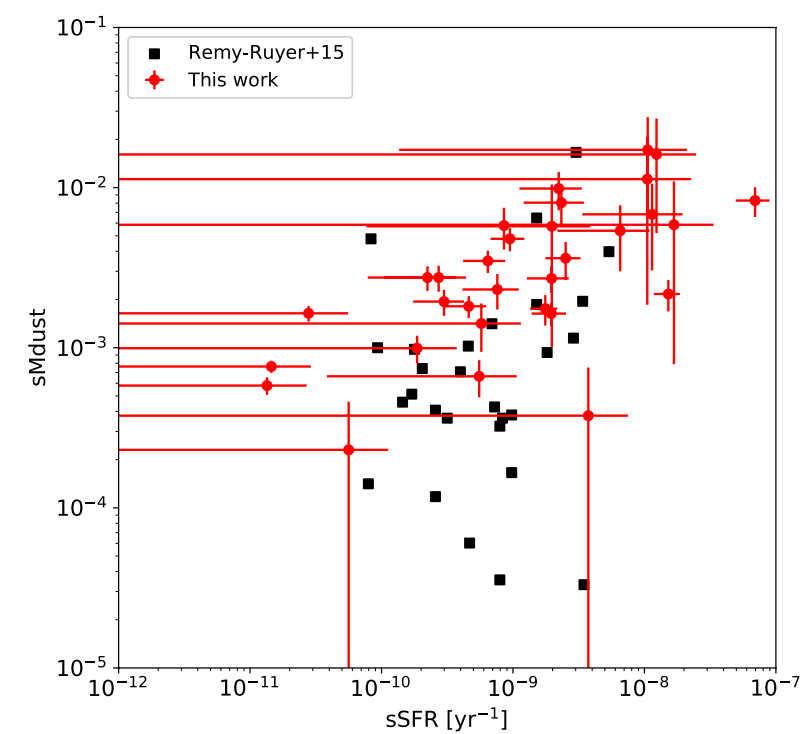

Fig. 3. sMdust vs. sSFR for the DGS galaxies selected and analysed in this work (red dots) and in Rémy-Ruyer et al. (2015) (black squares).

In Fig. 2 we show the comparison between the mass of dust, stellar content, and SFR derived in Burgarella et al. (2020) and those in Rémy-Ruyer et al. (2015). We find that the stellar mass and SFR derived by Rémy-Ruyer et al. (2015) are systematically larger than those derived in this work, while the mass of dust constrained by the IR emission is comparable. In Fig. 3 we compare the sMdust against the sSFR of the work by Rémy-Ruyer et al. (2015) with the ones derived from the SED fitting with CIGALE for the same galaxies. Our data points appear to be shifted at higher sMdust and a few of them present high sSFR. We discuss the implications of this for our analysis in the following section. The trend shown was derived through the SED fitting performed with a Chabrier IMF since a top-heavy one is not yet available in the SED fitting code CIGALE. By employing a top-heavy IMF in the SED fitting we do not expect significant variations in the stellar and dust masses derived, while the predicted age of the stellar population will tend to be older with lower values of sSFR (Pforr et al. 2012). Since the values of sMdust would remain approximately the same, we expect that the trend between sMdust and sSFR will still be present, but shifted to lower sSFR.

\subsection{Reproducing the gas, metal, and dust content of galaxies}

Here we present the results obtained from several tests from which we derive the suitable parameters to perform the systematic calculations and to build a grid of models through which we fit the properties of galaxies derived from CIGALE. The parameters adopted for these calculations and for computing the grid are listed in Table 2. We use different diagnostic diagrams such as sMdust, metallicity, and gas fraction as a function of the sSFR (or age) to investigate the metal and dust enrichment in galaxies, as well as the gas and dust removal from their ISM.

\subsubsection{Initial mass function and dust condensation fraction for $\mathrm{SNe}$ II}

The first constraint that we are able to add to our models is on the IMF, since not all the choices are able to reproduce the largest values of sMdust at large sSFR (corresponding to the beginning of the cycle). In the upper panel of Fig. 4 we show an example calculation adopting the Chabrier IMF and the top-heavy IMF
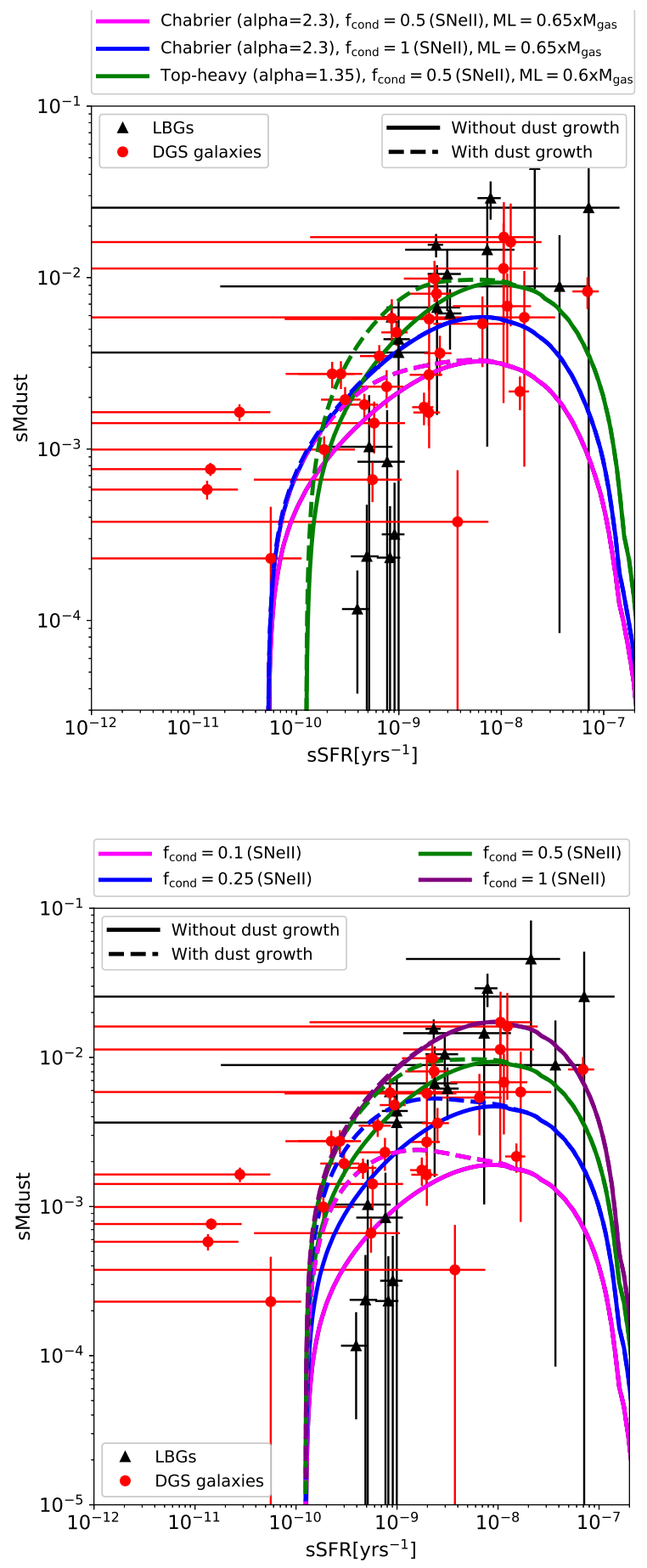

Fig. 4. Upper panel: sMdust against sSFR for LBGs (black triangles) and DGS galaxies (red dots). Different chemical evolutionary models computed with a Chabrier IMF or with a top-heavy IMF $(\alpha=1.35)$ and different condensation fractions as indicated in the legend. For all the models shown we select a value of $\tau=300 \mathrm{Myr}$ in Eq. (1) and $M_{\text {swept }}=1000 M_{\odot}$ in Eq. (8) and $M_{\text {gas }}=100 \times M_{\text {stars. }}$. Lower panel: same as in the upper panel but overplotting models with a top-heavy IMF and different condensation fractions indicated in the legend.

with $\alpha=1.35$. The cases with $\alpha=1,1.5$ yield results close to this latter case. We focus our investigation on models with $\tau=300 \mathrm{Myr}, M_{\text {gas }}=100 \times M_{\text {stars }}$, and $M_{\text {swept }}=1000 M_{\odot}$. Such a choice of parameters allows to us minimise the effect of dust destruction in the ISM (see Eq. (8)) which may prevent the models from attaining the largest values of sMdust at the 
beginning of the baryon cycle. For each of the models shown, the efficiency of the outflow reasonably reproduces the observations. Similar trends are recovered by employing $\tau=83 \mathrm{Myr}$. The observed values of sMdust at the beginning of the cycle are not well reproduced by a Chabrier IMF with $50 \%$ condensation fraction for $\mathrm{SNe}$. This holds in both cases where dust growth in the ISM is considered or neglected. The comparison improves when increasing the condensation fraction close to $100 \%$ for $\mathrm{SNe}$ which represents an extreme case, and still does not reproduce the sMdust for most of the galaxies at the beginning of the cycle. The observations are better reproduced if a top-heavy IMF is assumed. For this choice of the IMF, the ISM is rapidly enriched with metals and dust: a large value of sMdust $\left(\approx 10^{-2}\right)$ is attained within $100 \mathrm{Myr}\left(\approx \mathrm{sSFR} \approx 10^{-8} \mathrm{yr}^{-1}\right)$ by assuming a $50 \%$ condensation fraction for $\mathrm{SNe}$. The lower panel of Fig. 4 also shows the effect of changing the condensation fraction for SNe II. Even when dust growth in the ISM is included, a low condensation fraction of dust in $\mathrm{SNe}$ $(\approx 10 \%)$ prevents the models from reproducing the largest values of sMdust attained around sSFR $\approx 10^{-8} \mathrm{yr}^{-1}$. Therefore, a larger amount of dust needs to be condensed $(25-50 \%)$. In the same panel we also show the case with condensation fraction for $\mathrm{SNe}$ II close to the maximum. For this extreme value of the IMF and of the condensation fraction of dust, again the largest values of sMdust for some LBGs are not attained. This discrepancy between theoretical calculations and observations may depend on several factors, such as for example the chemical composition of dust assumed to derive the mass of dust from the far-infrared emission, which is affected by uncertainties of a factor of ten (Ysard et al. 2019).

In summary, a top-heavy IMF helps to reproduce the largest sMdust derived from observations. With this choice of IMF, a dust condensation fraction of around $50 \%$ is required. In this work, we consider condensation fractions between $25 \%$ and $\approx 100 \%$ for the systematic calculations.

\subsubsection{Gas reservoir and star formation efficiency}

For DGS galaxies it is possible to constrain the star formation efficiency (i.e. the mass of gas converted into stars) by studying the trend between their metallicity and the sSFR (or age). The comparison between model predictions and observations is shown in Fig. 5 where the metallicity $(\log (\mathrm{O} / \mathrm{H})+12)$ is plotted as a function of the sSFR. As a representative case, we study the behaviour of models with $\tau=300 \mathrm{Myr}, M_{\text {swept }}=$ $1000 M_{\odot}$, a condensation fraction of dust for SNe II of $50 \%$, a top-heavy IMF with $\alpha=1.35$, different mass-loading factors, and $M_{\text {gas }}=2-100 \times M_{\text {star }}$. The initial mass of baryons is composed of only gas at the beginning, which will then form stars according to the star formation law defined by Eq. (1). The quantity $(\log (\mathrm{O} / \mathrm{H})+12)$ is the abundance of oxygen in the gas phase, which is obtained in the models by subtracting the oxygen condensed into dust grains (silicates) and $\mathrm{CO}$ molecules from the total. From this plot we conclude that the initial mass of gas in the galaxy should be between 10 and 100 times the final stellar mass. For lower masses of gas, the ISM is enriched too quickly with respect to the observations. In the absence of outflow, the metallicity increases for a given choice of the mass of baryons until a plateau is reached. If some galactic outflow is present, the final metallicity attained is larger and rises more quickly. This is due to the fact that the outflow removes both metal and hydrogen from the ISM simultaneously but hydrogen is removed in larger amounts because its mass is larger than that of oxygen, and therefore the ratio decreases with time (see also Eq. (6)).

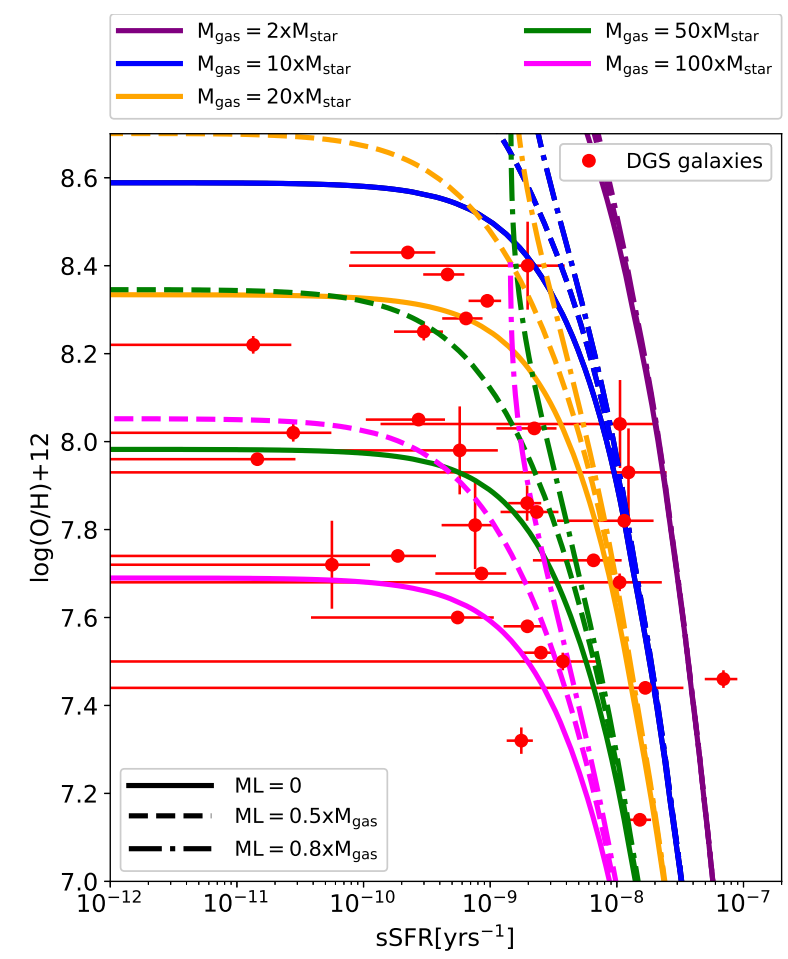

Fig. 5. Metallicity vs. sSFR for DGS galaxies (red dots). Different chemical evolutionary models characterised by diverse $M_{\text {gas }}$ and different mass-loading factors as mentioned in the legend are shown. For all the models, we select a value of $\tau=300 \mathrm{Myr}$ in Eq. (1), $\alpha=1.35$ for the top-heavy IMF in Eq. (3), and $M_{\text {swept }}=1000 M_{\odot}$ in Eq. (8), together with a dust condensation fraction for SNe II of $50 \%$. No dust growth in the ISM is considered.

Different combinations of initial baryon mass and outflow are able to cover the observed range of metallicity.

In summary, an initial gas reservoir between $\approx 10$ and $\approx 100$ times larger than the final stellar mass is needed to reproduce the observed metallicity of DGS galaxies. Different choices of the mass-loading factor allow us to cover the observed range of metallicity values. This constraint is used to set the input parameters for the evolution of LBGs for which the measurements of the metallicity are not available.

\subsubsection{Dust destruction by $\mathrm{SNe}$, star formation, and removal from galactic outflows}

After a peak in sMdust, a decline is observed for decreasing sSFR or increasing age, as shown in Fig. 1. In general, this effect can be due to a combination of (i) star formation, which increases the total stellar mass, (ii) dust astration by star formation, (iii) dust destruction from $\mathrm{SN}$ shocks, and (iv) galactic outflows. The two panels of Fig. 6 show the behaviour of different models computed with a top-heavy IMF, with the maximum value of $M_{\text {swept }}=6800 M_{\odot}$, and with different choices of the outflow for two extreme values of $M_{\text {gas }}=10 \times M_{\text {stars }}$ (upper panel) and $M_{\text {gas }}=100 \times M_{\text {stars }}$ (lower panel) selected as described in Sects. 5.2.2 and 5.2.1. From Fig. 6, it is clear that if outflow is not included, only in the case with the lowest mass of gas and no grain growth in the ISM does the combination of dust destruction from $\mathrm{SNe}$ and astration due to star formation partially reduce sMdust. In any case, all the models without outflow remain too flat for decreasing values of the sSFR. This trend indicates that dust astration and destruction 

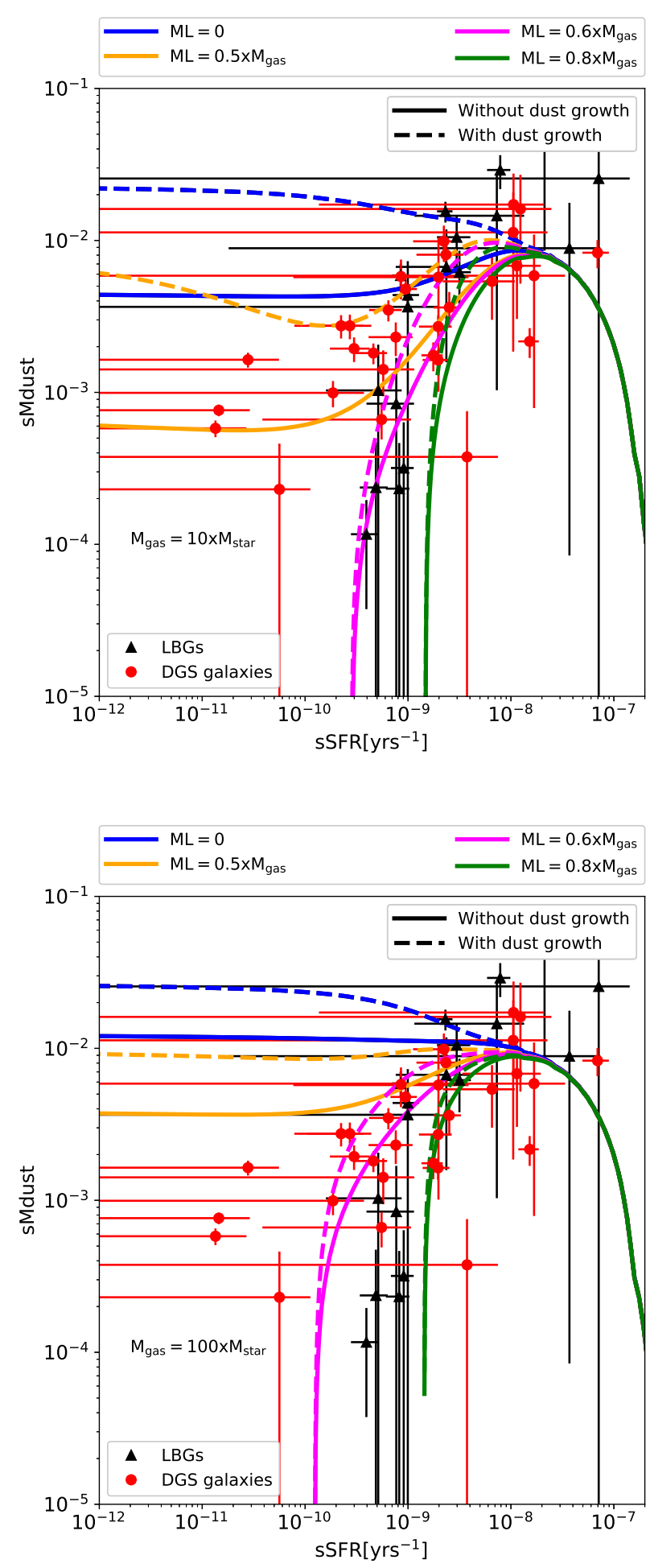

Fig. 6. sMdust vs. sSFR for LBGs (black triangles) and DGS galaxies (red dots) overplotted with different models with two extreme values of $M_{\text {gas }}$ : equal to $10 \times M_{\text {stars }}$ (upper panel) and to $100 \times M_{\text {stars }}$ (lower panel). Different combinations for the outflow efficiency are selected as mentioned in the legend. Both cases with and without dust growth in the ISM are shown. For all the models we select a value of $\tau=300 \mathrm{Myr}$ in Eq. (1), $\alpha=1.35$ for the top-heavy IMF in Eq. (3), and $M_{\text {swept }}=$ $6800 M_{\odot}$ in Eq. (8), together with a dust condensation fraction for $\mathrm{SNe}$ II of $50 \%$.

from $\mathrm{SNe}$ are not sufficient to decrease sMdust for decreasing SSFR, even for the maximum efficiency of dust destruction from

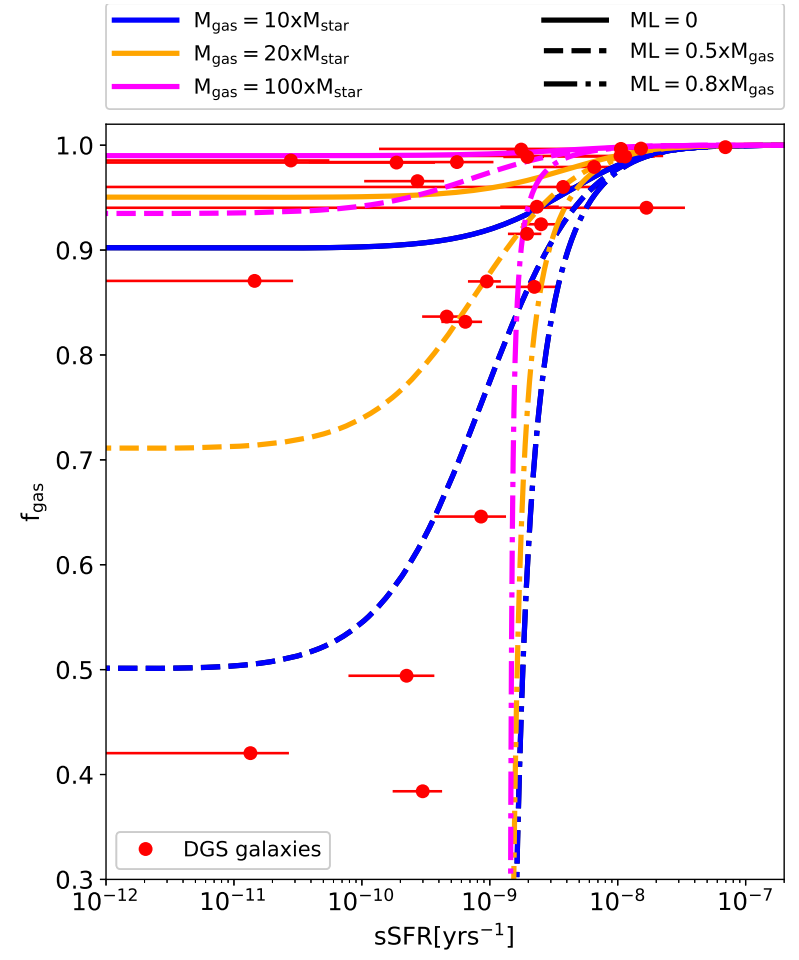

Fig. 7. Gas fraction vs. sSFR for DGS galaxies (red dots) overplotted with models with different choices of $M_{\text {gas }}$ and mass-loading factors. For all the models we select a value of $\tau=300 \mathrm{Myr}$ in Eq. (1), $\alpha=1.35$ for the top-heavy IMF in Eq. (3), and $M_{\text {swept }}=6800 M_{\odot}$ in Eq. (8), together with a dust condensation fraction for SNe II of 50\%.

SNe $\left(M_{\text {swept }}=6800 M_{\odot}\right)$. Indeed, since for most DGS galaxies a large amount of gas is required in order to be able to reproduce their metallicity trend, dust destruction by $\mathrm{SNe}$ is not efficient by construction (see Eq. (9)). By employing different values of $M L$ combined with $M_{\text {gas }}$ we are able to reproduce different sMdust in the galaxies. Therefore, we conclude that galactic outflows are required in order to reproduce the observed trend between sMdust and the sSFR for sSFR $<10^{-8} \mathrm{yr}^{-1}$ for both DGS galaxies and LBGs. We also notice that despite the fact that a combination with a mass of baryons equal to 100 times the final mass of the stars without galactic outflow will be suitable to reproduce the observed metallicity of galaxies with $\log (\mathrm{O} / \mathrm{H})+12<7.8$ and $\mathrm{sSFR}<10^{-9} \mathrm{yr}^{-1}$ the corresponding sMdust which is less than a few $10^{-3}$ at those sSFRs is not reproduced.

Galactic outflows are also needed in order to reproduce the observed gas fraction as a function of the sSFR for DGS galaxies. In Fig. 7 we show the observations for DGS galaxies overplotted with the evolutionary models characterised by different $M_{\text {gas }}$ and outflow efficiencies. In the case without outflow, only astration is at work in reducing the amount of available gas. As expected, gas consumption by star formation only mildly affects the gas fraction in cases where $M_{\text {gas }}=10 \times M_{\text {stars }}$, while the effect is negligible for larger gas content. Galactic outflows are therefore necessary to efficiently decrease gas fractions for decreasing sSFR.

In summary, galactic outflow is essential for reproducing the decline in sMdust for sSFR $\gtrsim 10^{-8} \mathrm{yr}^{-1}$ observed for both DGS galaxies and LBGs, and for reproducing the trend between the observed gas fraction and the sSFR in DGS galaxies. We therefore considered different efficiencies for this process in the systematic calculations. 
A. Nanni et al:: The gas, metal, and dust evolution in low-metallicity local and high-redshift galaxies

\subsubsection{Efficiency of dust growth in the ISM}

Here we discuss the efficiency of dust growth in the framework introduced in Sect. 3.3.3 in order to assess the relevance of this process in the galaxies under study. By looking at Figs. 4 and 6 it is possible to compare the efficiency of dust growth for different physical assumptions adopted in the simulations. In all the models shown, the chemical evolutionary tracks computed by including dust growth overlap with the ones without dust growth between sSFRs of $\approx 10^{-8} \mathrm{yr}^{-1}$ and $10^{-7} \mathrm{yr}^{-1}$, which corresponds to the early dust enrichment from SNe II. Around a sSFR of $\approx 10^{-8} \mathrm{yr}^{-1}$ the mass of dust tends to increase due to the effect of grain growth in the ISM. When the outflow is not included, the theoretical curves rise before reaching a plateau which corresponds to the maximum possible condensation of dust from the available metals (Fig. 6). This trend is qualitatively similar to the ones obtained by Asano et al. (2013). In the models without outflow, the increase of the dust in the theoretical tracks due to grain growth does not reproduce the observed decrease in sMdust, as already noticed for the models without grain growth. The models with and without dust growth become more similar for increasing efficiency of the outflow, which is an essential feature to reproduce the observed trends. In this case, the effect of grain growth in the ISM tends to be cancelled by dust removal. Furthermore, the effect of dust growth combined with a more efficient outflow is degenerate with the models without grain growth and less outflow.

From Fig. 4 we also notice that for low dust condensation fractions of SNe II and/or an IMF different from the top-heavy one, the galaxies with the largest sMdust observed at the beginning of the cycle are not reproduced even if grain growth in the ISM is included in the calculations. This might indicate that such a process is not the main one responsible for dust production at the beginning of the baryon cycle, unless we assume that grain growth occurs at larger densities $\left(>10^{3} \mathrm{~cm}^{-3}\right)$ or/and the dust grains initially present in the ISM are small $\left(<10^{-6} \mathrm{~cm}\right)$.

In summary, dust growth in the ISM is not dominant compared to outflow. Thus, it is not necessary to include it in order to reproduce the trend between sMdust and the sSFR for both DGS galaxies and LBGs. Thus, even in case dust growth were efficient, the effect of such a process may be cancelled by galactic outflows.

\subsubsection{Galactic inflows}

We here explore the possible effect of introducing galactic inflows in our models in addition to the outflow required to reproduce different observations. In the two panels of Fig. 8 we show as an example the effect introduced by including different amounts of inflow besides galactic outflow on the sMdust versus SSFR and on the metallicity versus sSFR plots for a few selected models with $\tau=300 \mathrm{Myr}, M_{\text {gas }}=50 \times M_{\text {stars }}$, $M_{\text {swept }}=6800 M_{\odot}$, and different choices of the galactic inflows and outflows. Since the dust and gas need to be removed from the galaxy, I/O in Eq. (5) needs to be $<1$. We select as test cases $\mathrm{I} / \mathrm{O}=0.2,0.5$ combined with efficient outflow $(\mathrm{ML}=$ $\left.0.6,0.8 \times M_{\text {gas }}\right)$. As shown in Fig. 8, in those models in which galactic inflow is included, an efficient outflow is needed in order to remove the dust in the galaxies and to reproduce the observations. The difficulty in removing the dust in the ISM in the presence of an inflow is caused by the fact that the dust is diluted in the ISM. This process decreases the dust fraction $\delta_{\mathrm{i}, \mathrm{d}}$ in Eq. (7) and dust is removed less efficiently from the galaxy. From the lower panel in the same figure, it is instead possible to see the slower metal enrichment due to the inflow of pristine gas, and
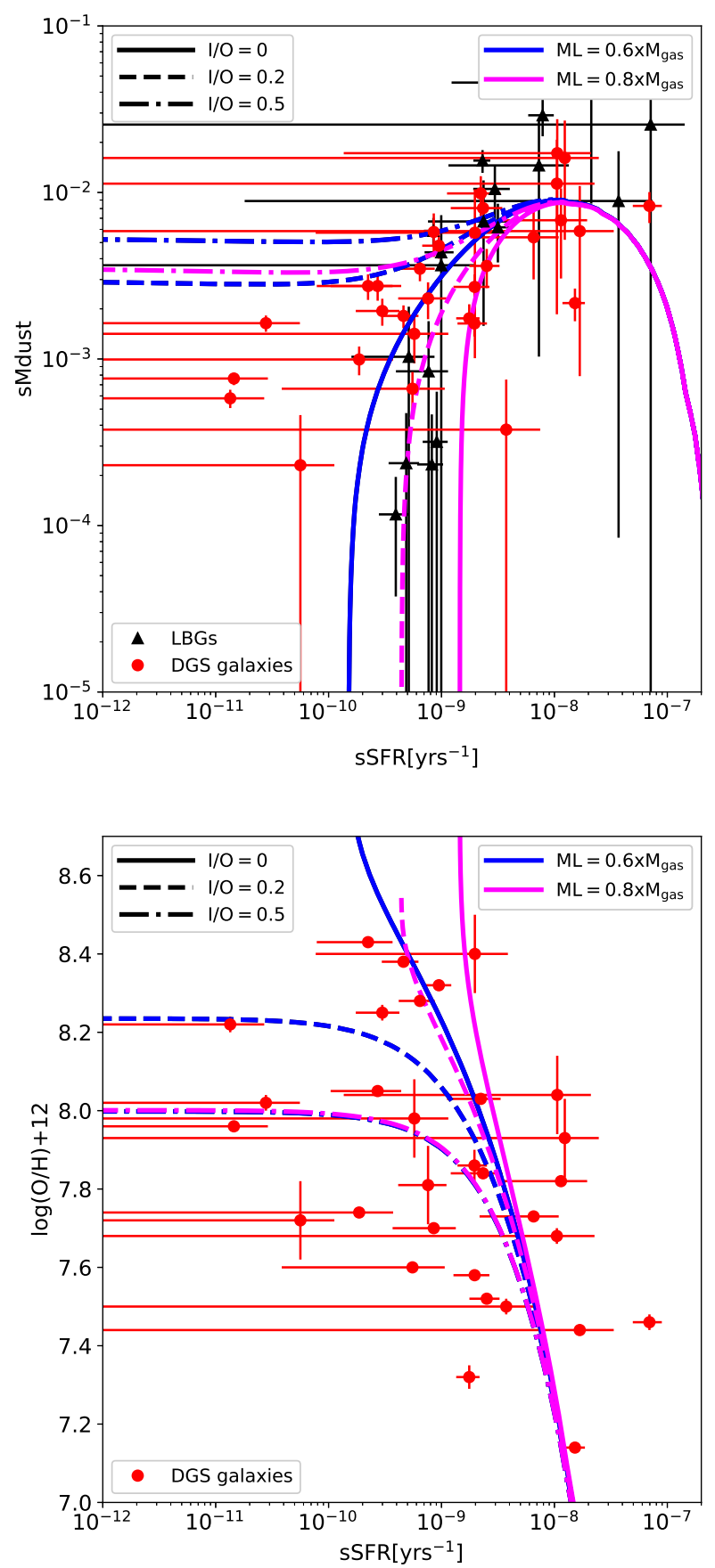

Fig. 8. Upper panel: sMdust vs. sSFR for LBGs (black triangles) and DGS galaxies (red dots) overplotted with models characterised by different choices of the mass-loading factor and inflows as indicated in the legend. For all models, we select a value of $\tau=300 \mathrm{Myr}$ in Eq. (1), $\alpha=1.35$ for the top-heavy IMF in Eq. (3), and $M_{\text {swept }}=6800 M_{\odot}$ in Eq. (8), $M_{\text {gas }}=50 \times M_{\text {stars }}$, and a dust condensation fraction for $\mathrm{SNe}$ II of $50 \%$. Lower panel: metallicity vs. sSFR for DGS galaxies overplotted with the same models as in the upper panel.

the corresponding decrease of the fraction of metals ejected in the outflow (due to the decrease of $f_{\mathrm{i}, \mathrm{g}}$ in Eq. (6)). Furthermore, the various combinations of galactic inflow and outflow introduce some degeneracy in the models with the ones discussed in the previous sections. We chose not to introduce any further degeneracy in our systematic calculations and to assume that all the gas is already present in the galaxy before the beginning of star formation. 


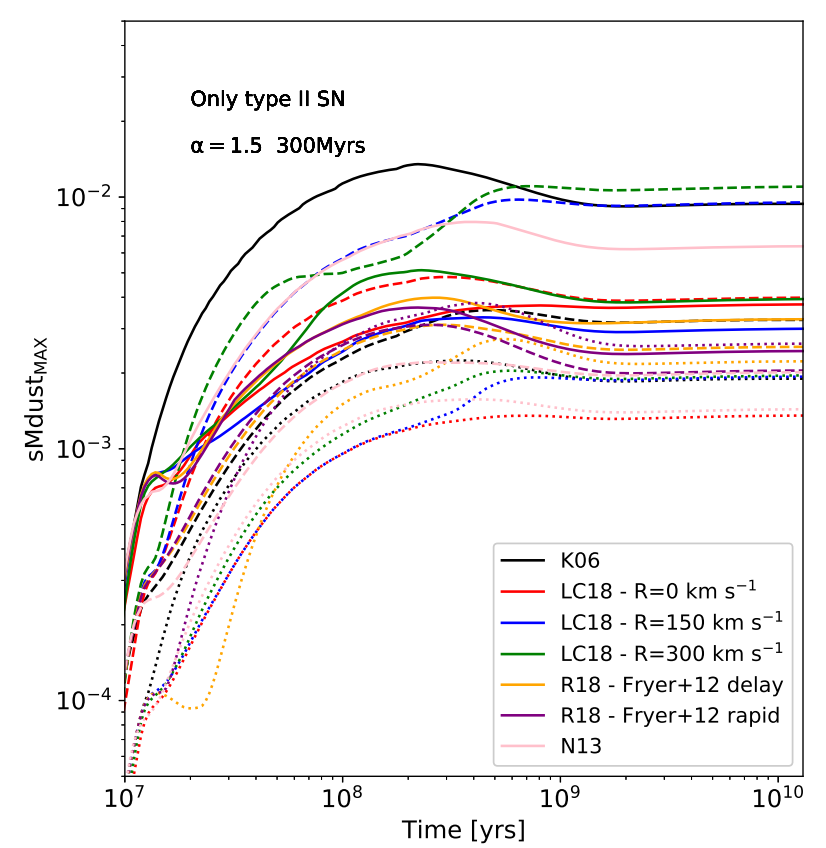

Fig. 9. Maximum possible sMdust for SNe II computed for different choices of the theoretical metal yields for massive stars listed in the figure. Solid, dashed, and dotted lines indicate the upper limits for sMdust obtained for silicate (only pyroxene), carbon, and metallic iron dust, respectively.

\subsubsection{Metals from different theoretical data sets and the contribution of different sources}

Here we explore the effect on chemical enrichment of employing different sets of theoretical metal yields taken from the literature, combined with the different timescales of the delayed SFH and top-heavy IMF. We consider seven different theoretical sets for SNe II, three for TP-AGB stars, and two for population III (pop III) stars (characterised by zero metallicity). For pop III stars, we assume the same IMF as for the other stars. The metal yields for SNe II by Kobayashi et al. (2006) and Nomoto et al. (2013) cover a mass range up to $40 M_{\odot}$. Limongi \& Chieffi (2018) provide three sets of metal yields up to $120 M_{\odot}$ computed for different rotational velocities, $R$, of massive stars $(R=0$, $150,300 \mathrm{~km} \mathrm{~s}^{-1}$ ). Ritter et al. (2018) computed two sets of metal yields by employing different formalisms up to $25 M_{\odot}$. The properties of the different data sets are summarised in Table 2.

Starting from these metal yields we calculate the maximum possible amount of dust produced considering silicates, carbon, and iron dust. For simplicity, we consider the case in which silicate dust is composed entirely of pyroxene $\left(\mathrm{MgSiO}_{3}\right)$. However, the mass of silicates derived if only olivine $\left(\mathrm{Mg}_{2} \mathrm{SiO}_{4}\right)$ is considered does not change considerably. For silicates, the maximum possible mass of dust is estimated at each time-step by evaluating the least abundant of all the elements that form this dust species. The final mass of dust is then obtained by estimating the maximum number of monomer and mass of dust that can be formed from the stoichiometric formula of pyroxene.

A representative example of the results for $\mathrm{SNe}$ II is shown in Fig. 9 where the maximum possible sMdust of silicate, carbon, and iron dust is plotted as a function of time. A top-heavy IMF with $\alpha=1.5$ and $\tau=300 \mathrm{Myr}$ has been selected. The trend obtained by employing different data sets is qualitatively similar for different IMF and $\tau$. The spread in the maximum sMdust spans a factor of about five for all the dust species considered. For all the data sets except that of Limongi \& Chieffi

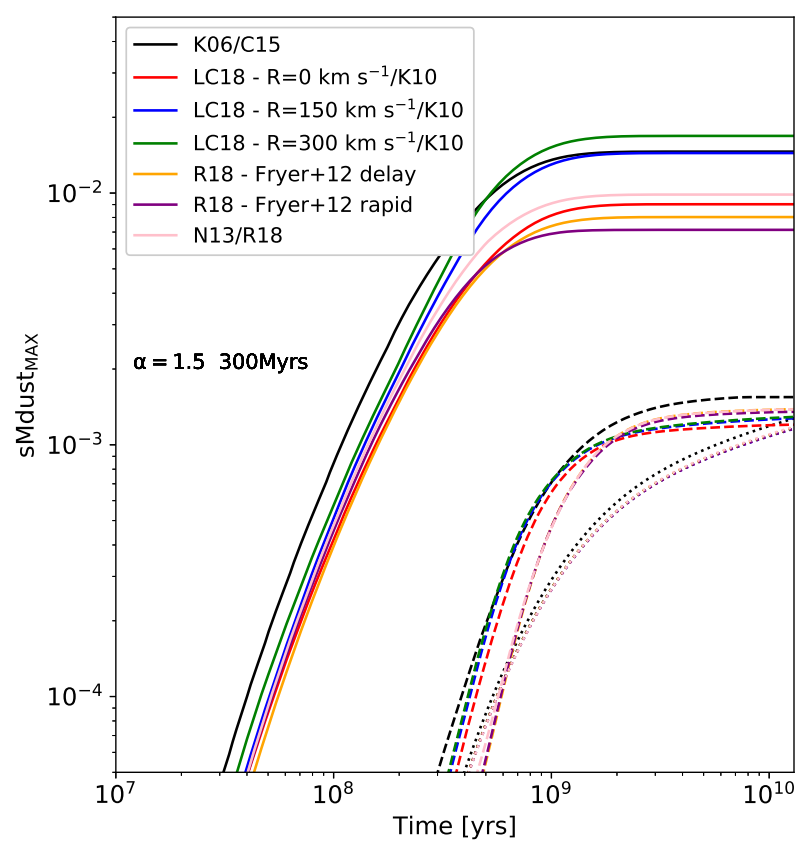

Fig. 10. Maximum possible sMdust for SNe II (solid lines), TP-AGB stars (dashed lines), and Type Ia SNe (dotted lines) for different choices of the theoretical metal yields for SNe II and TP-AGB stars listed in the figure and provided in Table 2.

(2018), the final mass of silicate dust is larger than carbon by a factor between 1.2 and 3.6. For the three data sets by Limongi \& Chieffi (2018), the mass of silicate dust is down to one-third the mass of carbon dust. The maximum iron mass is around $10 \%$ and $15 \%$ of the total dust mass for Kobayashi et al. (2006) and Limongi \& Chieffi (2018), and for Nomoto et al. (2013), respectively. For the two data sets by Ritter et al. (2018) the mass of iron dust is between 30 and $35 \%$ of the total. The sets of models that produce the largest maximum mass of dust are from Kobayashi et al. (2006) and Limongi \& Chieffi (2018) with rotational velocity $R=150,300 \mathrm{~km} \mathrm{~s}^{-1}$. The three data sets yield very similar values of sMdust from SNe II close to the expected observed peak of dust around 100-200 Myr. The chemistry of dust is dominated by silicates if the chemical yields by Kobayashi et al. (2006) are employed or by carbon dust if the ones by Limongi \& Chieffi (2018) with $\mathrm{R}=150,300 \mathrm{~km} \mathrm{~s}^{-1}$ are adopted. For Kobayashi et al. (2006), the maximum amount of silicates produced is three times larger than carbon dust. The differences between the dust chemistry of the ISM obtained by adopting different theoretical yields might be compared and constrained with future observations. For these three data sets, we also checked the amount of oxygen released into the ISM, as oxygen is the most abundant metal and traces the metallicity of galaxies. The predicted oxygen abundances are compared with observations. We find that the values are always comparable even for different $\tau$ and for the different top-heavy IMF. Furthermore, since reproducing the large mass of observed dust in the Early Universe is challenging, we expect the comparison with observations to worsen by employing the theoretical data sets yielding a lower amount of metals and dust with respect to Kobayashi et al. (2006) and Limongi \& Chieffi (2018).

To test the effect on the yields from TP-AGB stars we consider different combinations of theoretical yields for SNe II and TP-AGB stars. The chemical enrichment from TP-AGB stars is affected by the choice of the SN theoretical yields that change the metallicity of low-mass stars as a function of time and 
therefore the overall metal and dust masses released in the ISM. The combinations considered are provided in Table 2 . The results are shown in Fig. 10 where we plot the maximum possible sMdust for SNe II, TP-AGB stars, and SNe Ia. For all the combinations of yields, we find that the contribution from TP-AGB stars and SNe Ia is negligible due to the top-heavy IMF. Furthermore, in cases where a larger amount of dust were released by TP-AGB stars or SNe Ia, it would start to be relevant only after a few hundreds million years, when the dust is expected to start to decrease in the observed galaxies. A comparable amount of sMdust could be obtained for TP-AGB stars and SN remnants by assuming a drastically low condensation efficiency in SN remnants, but this is not our preferred scenario, as discussed in the previous sections. Indeed, the contribution from TP-AGB stars is more relevant where the selected IMF is the Chabrier one. However, this scenario is never able to reproduce the observed trends for the galaxies considered in this work.

We additionally tested two different theoretical yields for Pop III stars (Heger \& Woosley 2010; Nomoto et al. 2013). We find that the contribution to the metal enrichment of Pop III stars is negligible with respect to the total.

In summary, the metal yields selected throughout this paper for the systematic calculations are from Kobayashi et al. (2006) since these allow for the most favourable conditions for attaining the largest values of sMdust already after $100 \mathrm{Myr}$ from the beginning of the baryon cycle. The choice of the metal yields for Pop III, TP-AGB stars, and Type Ia SNe does not largely affect the results, since these stellar sources provide only a minor contribution to the dust enrichment. We therefore arbitrarily select the theoretical yields by Heger \& Woosley (2010) for Pop III stars (at the ages appropriate for the sample), Cristallo et al. (2015) for TP-AGB stars, and Iwamoto et al. (1999) for Type Ia SNe.

\subsubsection{Input quantities for the systematic calculations}

On the basis of the tests discussed in the previous sections, we are able to define the characteristics required to systematically compute the chemical evolutionary models which are then used to perform the best fit between model predictions and observations:

- Metal yields favouring a fast metal enrichment from SNe II (e.g. Kobayashi et al. 2006);

- top-heavy IMF;

- total mass of baryons $\geq 10$ times the final stellar mass;

- efficient galactic outflow;

- condensation fraction for dust produced in SNe II $\geq 25 \%$;

- no requirement for dust growth in the ISM.

The input quantities adopted to compute the grid of models are summarised in Table 2 (referred to there as "Systematic calculations").

\subsubsection{Characterisation of the individual galaxies}

Following the procedure described in Sect. 4, we estimate the properties of DGS galaxies and LBGs, such as the chemical composition of their dust, the fraction between the gas and dust ejected into the circumgalactic medium, and the fraction of dust destroyed by SN shocks. In Figs. 12-15 we show the different properties of galaxies derived from the SED fitting or taken from the literature, i.e. sMdust, metallicity, gas fraction, and dust-togas ratio, as a function of the sSFR and age together with the corresponding distribution obtained from the fit of individual objects through the models in the chemical evolutionary tracks.
In Figs. 13-15 we also show the metallicity, gas fraction, and dust-to-gas ratio as a function of the sSFR and time for LBGs, for which reliable constraints are not yet available. We additionally show the dust-to-gas ratio as a function of the metallicity in Fig. 16. For the DGS galaxies, this trend is compared with the available estimates, while for LBGs only the values derived from the chemical evolution models are shown. In Fig. 17 we show and the total mass of dust inside the galaxy versus the mass of stars.

The best fits between the properties of individual galaxies and model predictions are provided in Table 3. The complete version of the table is provided at the CDS. From the performed analysis we derive the following trends:

Overall properties. The models developed here are in general able to fit the main properties of the observed DGS galaxies and LBGs. The stellar production efficiency (defined as the mass of stars over the total mass of initially available baryons) is usually of few percent for both DGS galaxies and LBGs. A strong outflow is required in order to remove the mass of dust and gas present in the galaxy and to reproduce the observation both for DGS galaxies and LBGs. Depending on the specific galaxy under consideration, the amount of gas removed from the ISM ranges between $\approx 6-97 \%$ for DGS galaxies and $\approx 12$ and $96 \%$ for LBGs. The exponent of the IMF in Eq. (3) is $\alpha \approx 1$ for the majority of the DGS galaxies, while this value is not well constrained for LBGs for which the results are more degenerate. This is not surprising because there is less information available for LBGs. The set of observations for DGS galaxies is reproduced by a condensation fraction of SNe II which is between $25 \%$ and $64 \%$ with an average value of $(35 \pm 12) \%$ considering the entire sample of DGS, while for LBGs a condensation fraction above $40 \%$ is required with an average value of $56 \pm 17 \%$ if all the LBGs are considered. Also in this case, a larger uncertainty on the values of the condensation fraction is found for LBGs. We also keep in mind that there is some degeneracy between the metal yields provided in the literature and the assumed dust condensation fraction.

The dust-to-gas ratio as a function of the metallicity is fairly well reproduced for DGS galaxies (Fig. 16), even though the observed distribution of data points is more tilted than the one we recovered. This discrepancy between our model predictions and observations might be partially due to the large uncertainties affecting the determination of the gas mass from the observations, especially in low-metallicity galaxies. The trend between the total mass of dust and stellar mass is naturally reproduced (Fig. 17).

Dust chemical composition and condensation fraction. The predicted chemical composition of dust changes as a function of time. This is determined by the IMF and by the condensation fractions assumed for the different species. The dust chemical composition is dominated by silicates which are always between 65 and $80 \%$ of the total mass fraction. Carbon dust mass is instead a factor of between 0.6 and 1.6 of the mass of iron. We are aware of the fact that such chemical composition of dust is expected to be dependent on the metal yields selected for SNe II.

Efficiency of the outflow and of SN shocks for dust removal. We estimate the amount of dust removed from the ISM through galactic winds and destroyed by SN shocks for each of the galaxies considered. Depending on the galaxy, between $\approx 20 \%$ and $\approx 90 \%$ of the dust disappearing from the ISM is removed by galactic outflows rather than being destroyed by SN shocks. 


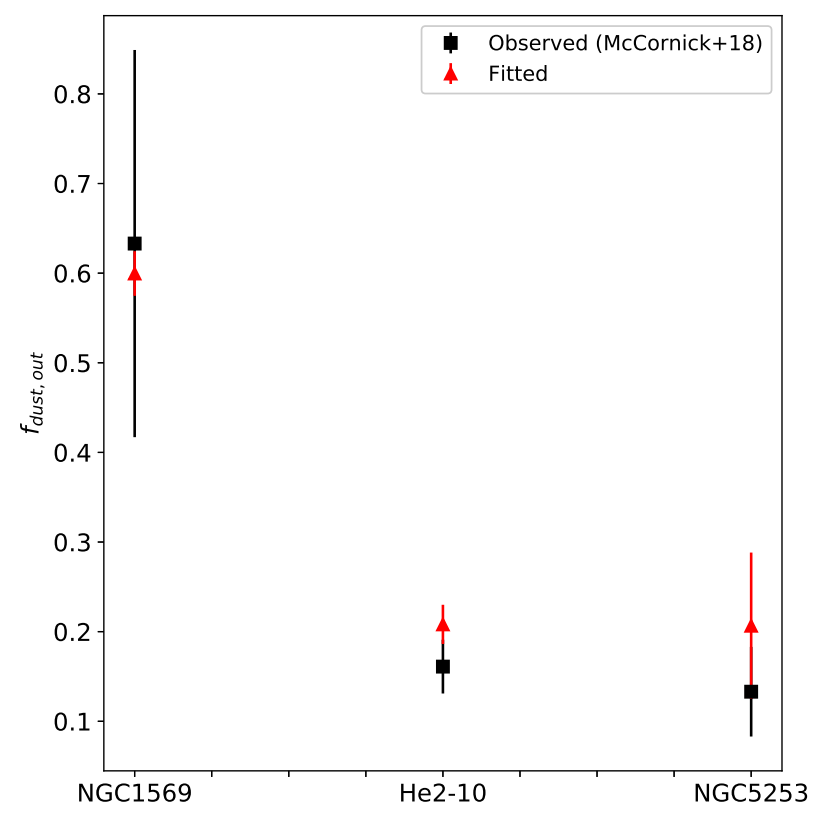

Fig. 11. Circumgalactic dust mass fraction from McCormick et al. (2018) (black squares) compared with the predicted value derived from the fit of each galaxy (red triangles).

For three of the DGS galaxies in our sample (NGC 1569, He2-10, NGC 5253), estimates of circumgalactic dust are available from McCormick et al. (2018). We use this information to fit the galaxies to the models; the observed and predicted distributions are shown in Fig. 11. For the three galaxies considered, our approach provides a satisfactory fit of the observed circumgalactic dust fraction.

Comparison between DGS galaxies and LBGs. The metallicity values and gas fractions predicted by our models for LBGs are comparable with the ones derived for DGS. However, we predict a faster metal enrichment for the LBGs than for DGS galaxies (lower panel of Fig. 13) and the gas is ejected on a shorter timescale for LBGs $(\approx 400 \mathrm{Myr})$ than for DGS galaxies $(\approx 1 \mathrm{Gyr})$. Figure 15 shows that the typical dust-to-gas ratio of LBGs is comparable with the upper limit observed for DGS galaxies as a consequence of the large condensation fraction required to explain the sMdust of these galaxies.

\section{Discussion}

In the present work we show that a top-heavy IMF and high condensation fraction in $\mathrm{SN}$ remnants are required in order to have a fast increase of the dust content in the ISM of galaxies within $100 \mathrm{Myr}$ from the beginning of the baryon cycle. This finding is in agreement with other works in the literature in which the large amount of dust in Quasars at $z>6$ is explained by adopting similar assumptions (Gall et al. 2011a,b). This result is also in agreement with the work from Liu \& Hirashita (2019) who find that most of the ALMA observations of galaxies at $z>7$ can be explained by assuming a dust condensation fraction of $\approx 0.5$ for stellar sources. In the local Universe, top-heavy IMFs have been adopted to explain some observational properties of ultra-compact dwarf galaxies (Dabringhausen et al. 2012), ultrafaint dwarf galaxies (Geha et al. 2013; McWilliam et al. 2013) and Galactic globular clusters (Marks et al. 2012).

On the other hand, in many works in the literature dust growth in the ISM has been considered to be of fundamen-
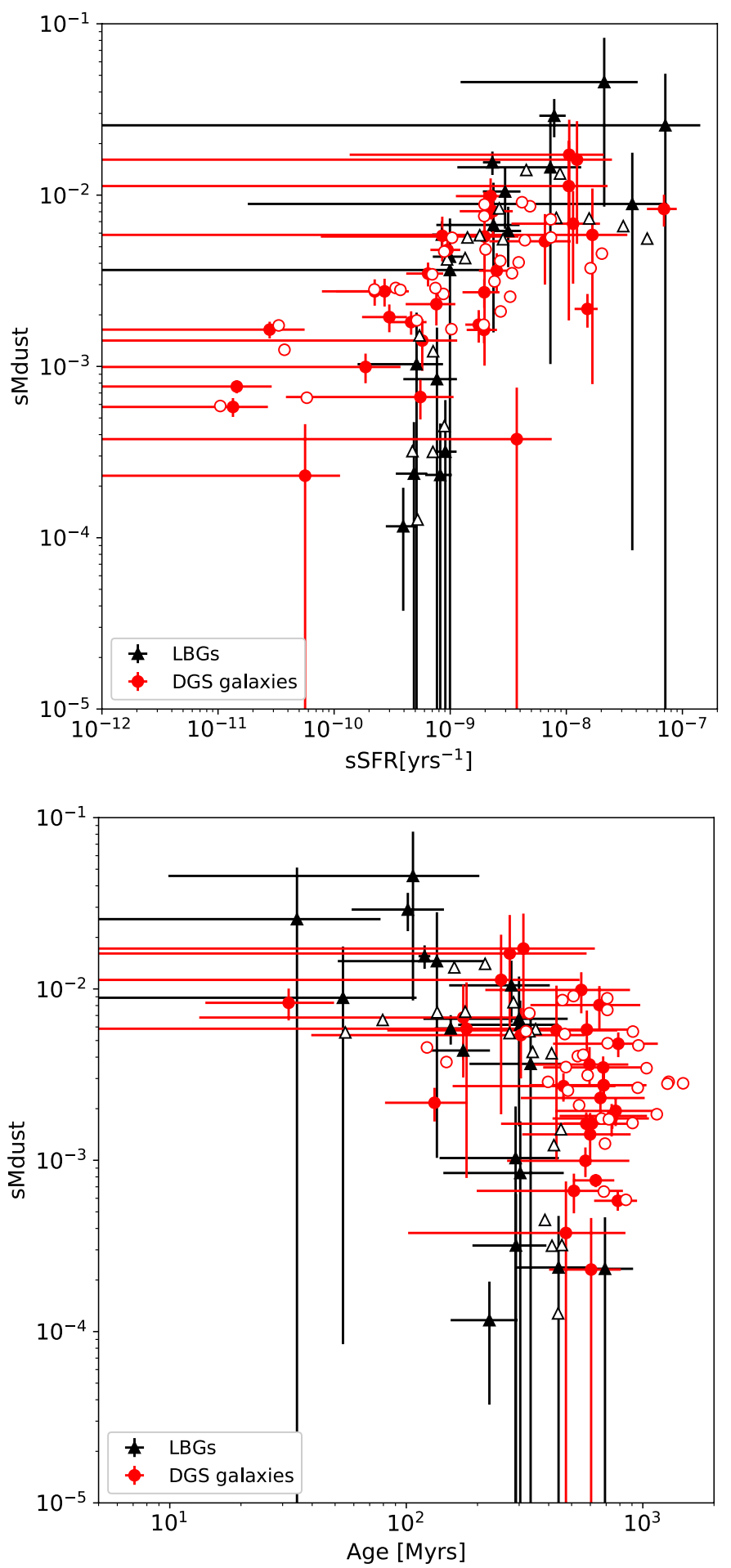

Fig. 12. Upper panel: sMdust as a function of sSFR for LBGs derived from the SED fitting with CIGALE (full black triangles) and DGS galaxies (full red dots) overplotted with the distribution obtained from the likelihood analysis of chemical tracks described in Sect. 4 (empty symbols). Lower panel: sMdust as a function of age, colour coded as in the upper panel.

tal importance to explain the amount of dust observed in local and high-redshift galaxies (Asano et al. 2013; Zhukovska 2014; Michałowski 2015; Aoyama et al. 2017; Ginolfi et al. 2018; Leśniewska \& Michałowski 2019). Popping et al. (2017) predicted efficient dust growth in the ISM for galaxies with stellar masses $\log \left(M_{*} / M_{\odot}\right)>7$, while dust enrichment from 
A. Nanni et al.: The gas, metal, and dust evolution in low-metallicity local and high-redshift galaxies
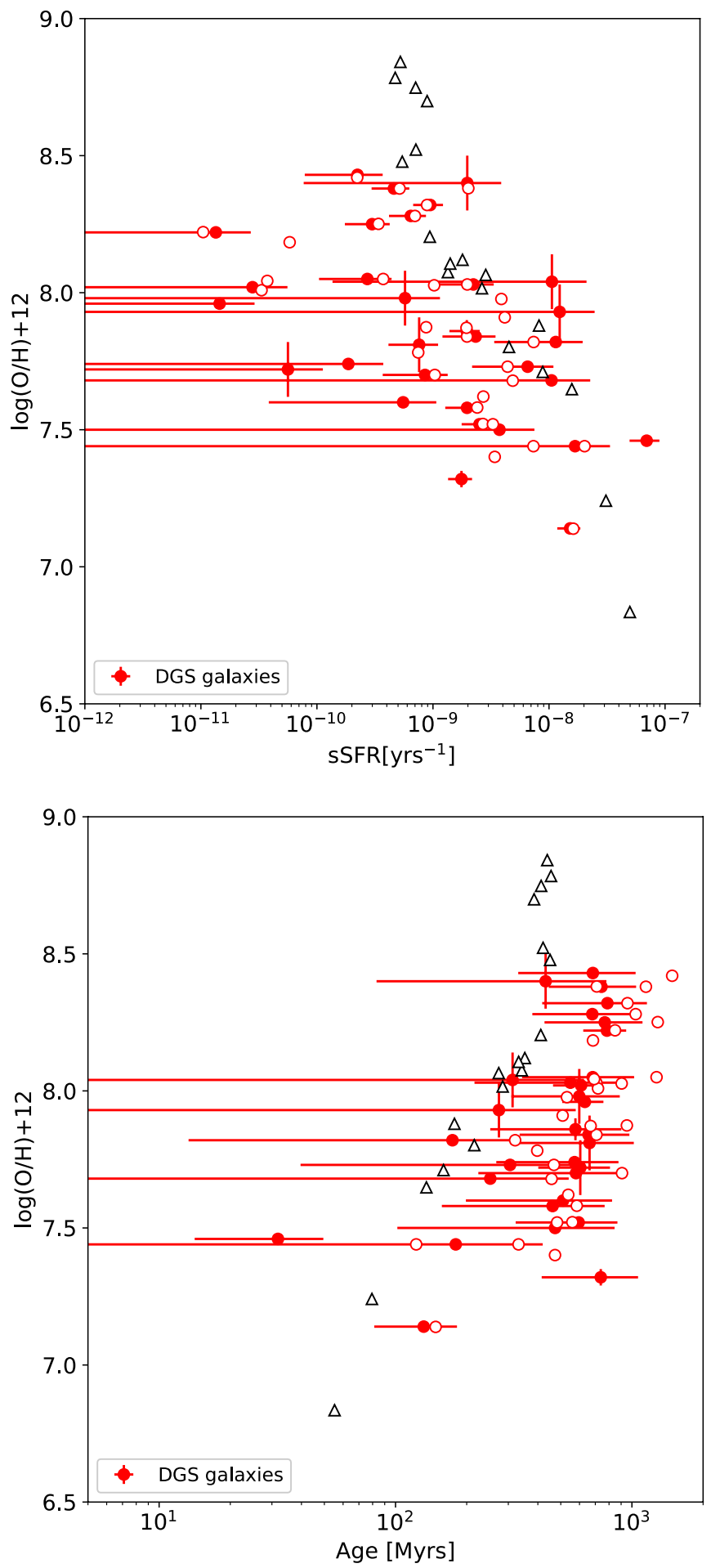

Fig. 13. Upper panel: metallicity as a function of sSFR. Lower panel: metallicity as a function of age. The same colour-coding is adopted as in Fig. 12 . The predicted distribution for LBGs is shown for comparison.

stellar sources appears to be dominant for galaxies characterised by lower masses. Mancini et al. (2015) and Graziani et al. (2020) found a similar trend but for larger stellar masses of $\log \left(M_{*} / M_{\odot}\right)>9$ and $8.5<\log \left(M_{*} / M_{\odot}\right)<9.5$, respectively. The dependence between the efficiency of grain growth in the ISM and the stellar mass of the galaxies found in these latter studies is ascribed to the presence of more metals in more massive galaxies. Schneider et al. (2016) also pointed out the depen-
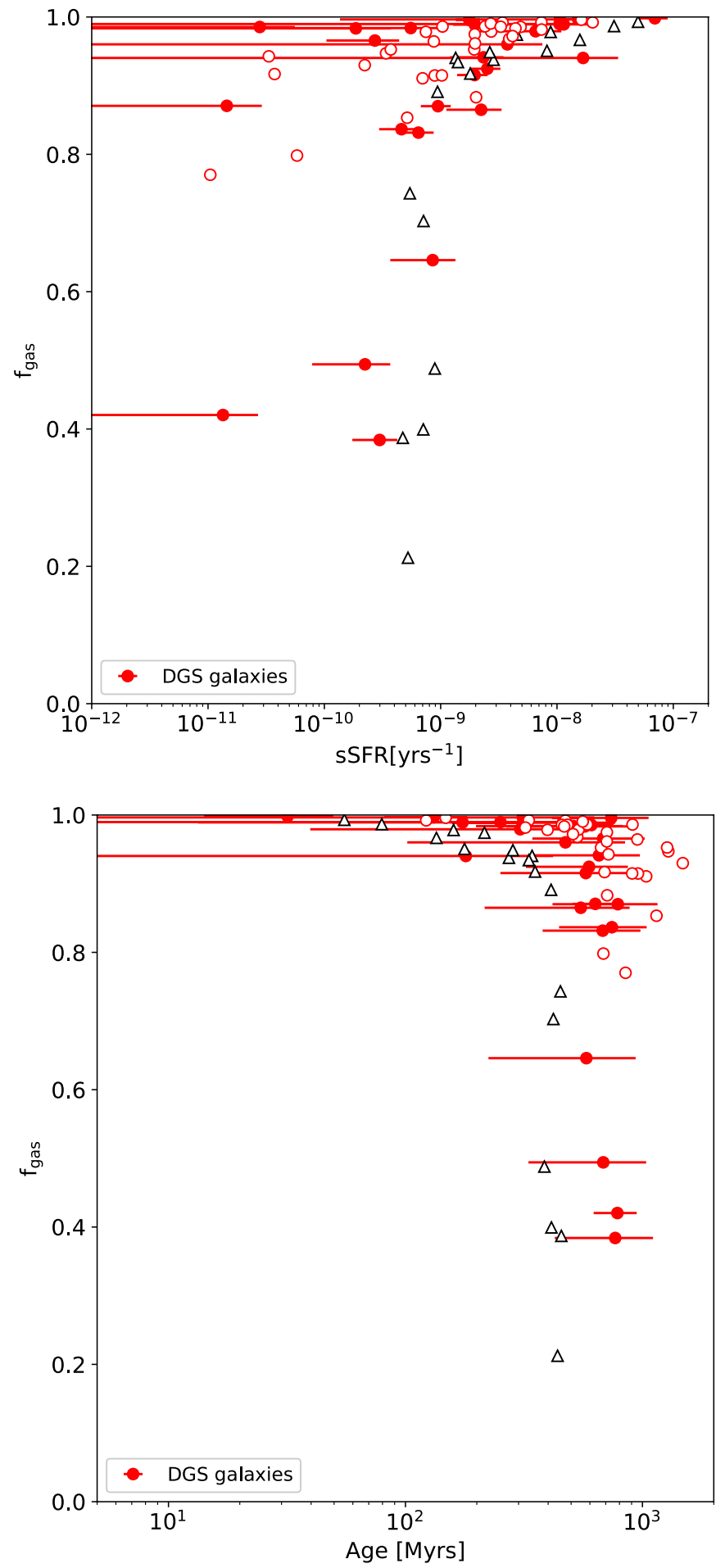

Fig. 14. Upper panel: gas fraction as a function of sSFR. Lower panel: gas fraction as a function of age. The same colour-coding is adopted as in Fig. 12. The predicted distribution for LBGs is shown for comparison.

dence of grain growth efficiency with the density of the gas in the galaxy. da Cunha et al. (2010) use the model from Calura et al. (2008) for dwarf galaxies which includes grain growth in the ISM, and in which a Salpeter IMF is adopted for these galaxies. However, the galaxies at larger sMdust and sSFR are not reproduced by the da Cunha models (see Fig. 10, left panel of da Cunha et al. 2010). This result is not in contradiction with our analysis, since we need to assume a top-heavy IMF in order 

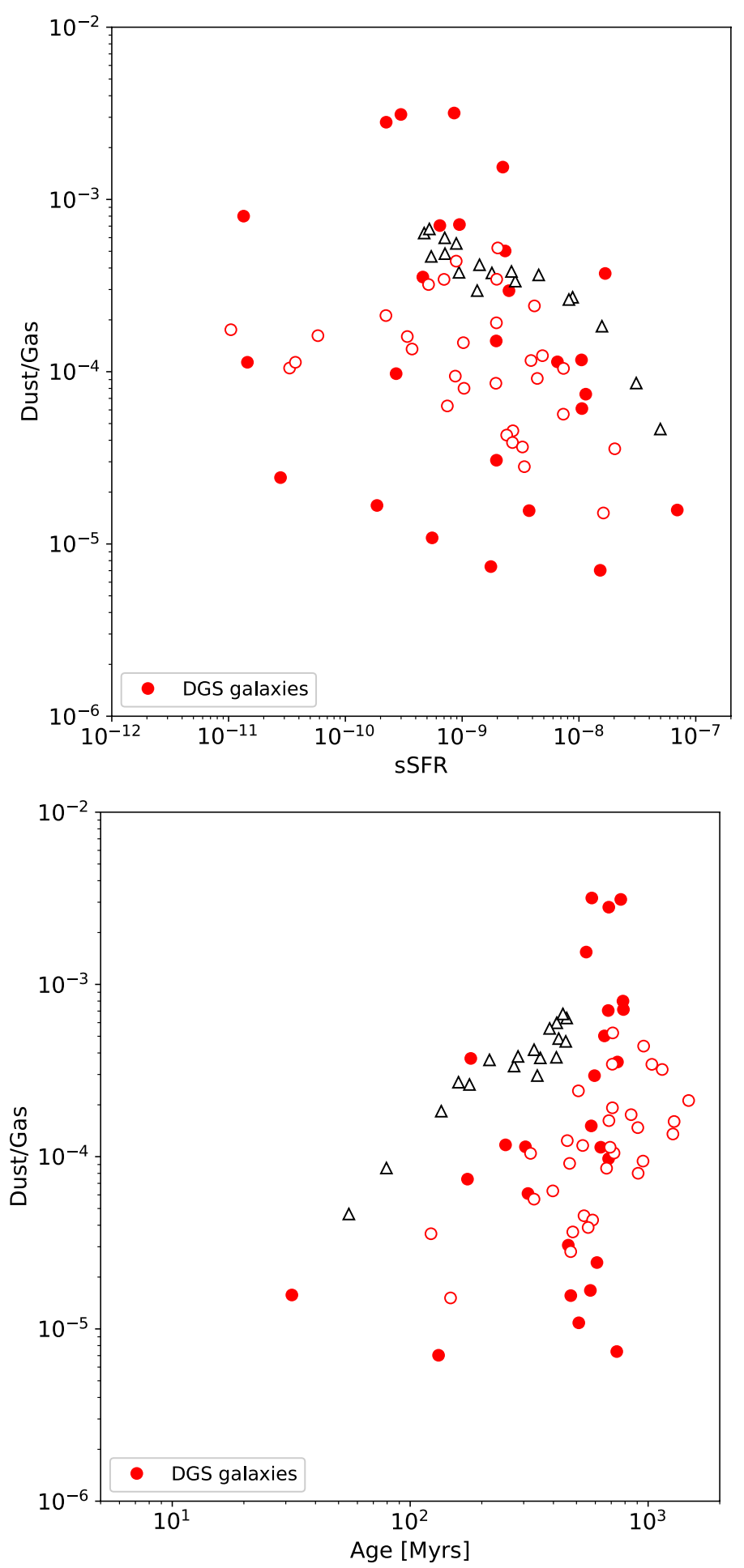

Fig. 15. Upper panel: dust-to-gas ratio as a function of sSFR. Lower panel: dust-to-gas ratio as a function of age. The same colour-coding is adopted as in Fig. 12. The predicted distribution for LBGs is shown for comparison.

to reproduce the larger values of sMdust and this option was not explored by these latter authors.

In the analysis performed by Rémy-Ruyer et al. (2015) the models developed by Asano et al. (2013) are employed. As shown in Fig. 11 of Rémy-Ruyer et al. (2015), the sMdust of galaxies with sSFR between $10^{-9}$ and $10^{-8}$ is not reproduced. Following the analysis by Rémy-Ruyer et al. (2015),

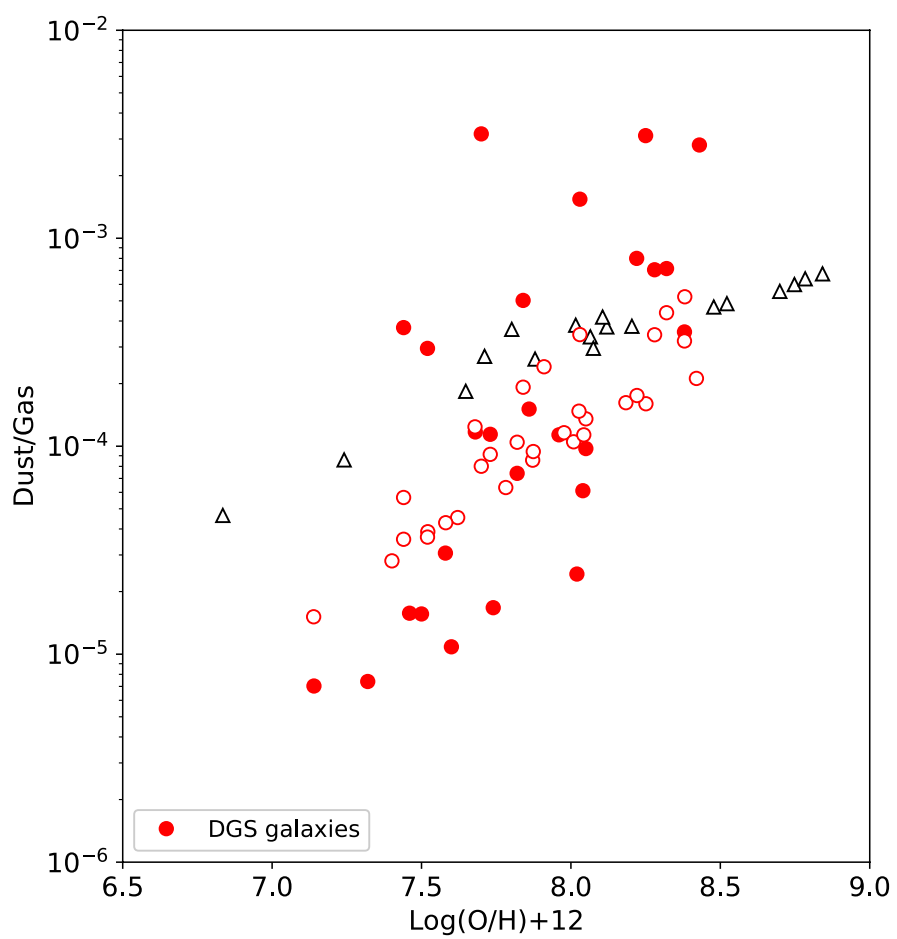

Fig. 16. Observed and predicted dust-to-gas ratio as a function of metallicity. The same colour-coding is adopted as in Fig. 12. The predicted distribution for LBGs is shown for comparison.

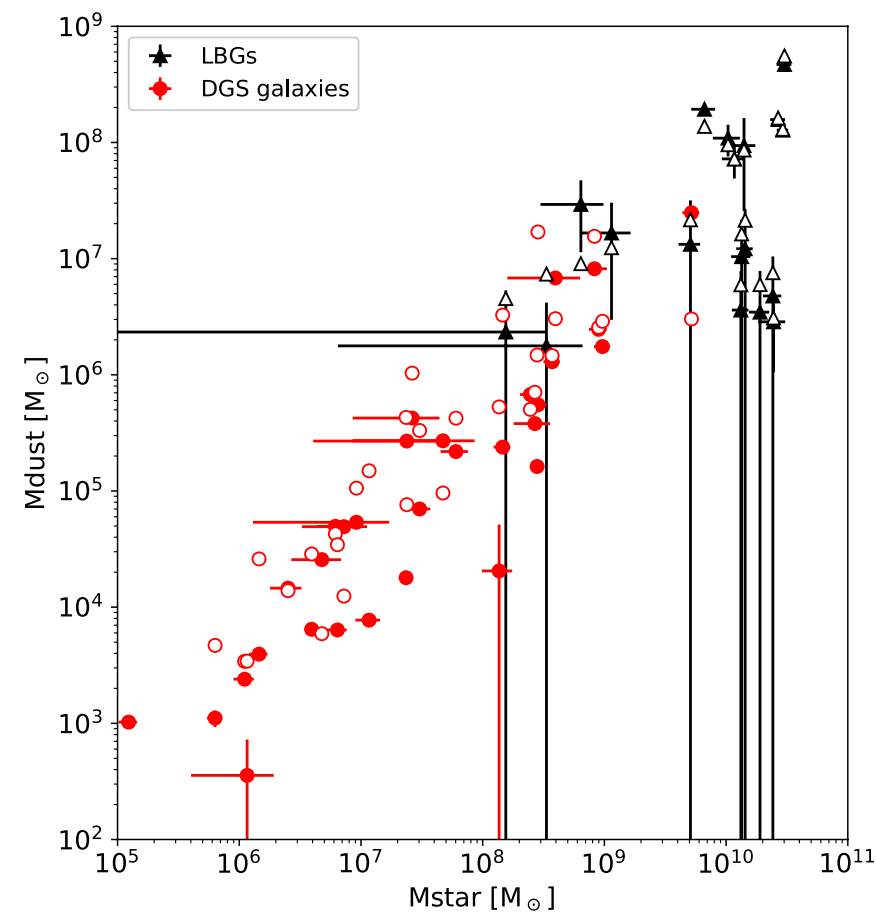

Fig. 17. Observed and predicted mass of dust as a function of the stellar mass. The same colour-coding is adopted as in Fig. 12.

De Vis et al. (2017, 2019) reinterpreted the properties of the same sample of galaxies by studying their chemical evolution under different scenarios (e.g. with and without dust growth in the ISM). These latter authors obtained their best-fit models and the properties of DGS derived by Rémy-Ruyer et al. (2015) by employing a Chabrier IMF and assuming: (a) a star formation history characterised by several bursts as proposed by 
Zhukovska (2014), (b) that dust can grow in the ISM but at lower rates with less efficient star formation, and (c) a stronger inflow and outflow in DGS galaxies than in less star-forming galaxies. Despite the ability of such a model to reproduce the overall observed trends of different samples of local galaxies, the DGSs characterised by the largest SSFR remain challenging to explain (see the top-left panel of Fig. C1 in De Vis et al. (2017)). The tension between this kind of model and observations is even more critical in our analysis since our SED fitting yields values of the sMdust at earlier epochs (larger sSFR) that are even larger than the ones obtained by Rémy-Ruyer et al. (2015) and employed by De Vis et al. (2017, 2019), as discussed in Sect. 5.1. Such a difference implies that larger values of sMdust should be achieved in DGS galaxies earlier than in the aforementioned works. In our work, this effect is obtained by adopting a top-heavy IMF which increases the amount of metals available to form dust already at early epochs coupled with a condensation fraction $>25 \%$ for SNe II. In such a framework, dust growth in the ISM is not needed to produce the total amount of dust observed. Moreover, we show that this process would become efficient when the galactic outflow is expected to start to reduce the amount of gas and dust in the galaxies. Therefore, even if present, the outflow would cancel the effect of dust growth in the ISM.

The trend between dust-to-gas ratio and metallicity is well reproduced for DGS galaxies by Zhukovska (2014) who included dust growth in the ISM and a very low condensation fraction for silicates in her calculations $\left(f_{\text {sil }}=10^{-3}\right)$. The same observed trend is not well reproduced by assuming a more efficient condensation in SN remnants and no dust growth in the ISM, similarly to what we derive in Fig. 16. Contrary to our work and to the analysis by De Vis et al. $(2017,2019)$ a closedbox model was employed in Zhukovska (2014) together with a variety of SFH for the galaxies. However, the comparison with the observations is limited to the trend between the dust-to-gas and metallicity. In our analysis, the low condensation fraction from $\mathrm{SNe}$ II required to reproduce this trend does not reproduce the large value of sMdust at the beginning of the cycle. The discrepancy between our model predictions and observations might be partially due to the large uncertainties in the determination of the mass of gas in low-metallicity galaxies.

Ginolfi et al. (2018) also proposed dust growth in the ISM of dwarf galaxies to explain the observed amount of dust. However, the investigation is limited to a Salpeter IMF, and the possibility of producing a larger amount of dust by employing a top-heavy IMF is not explored in their work.

By exploring the microphysics of grain accretion, Ferrara et al. (2016) indicated that such a process in the diffuse ISM is likely to be inefficient due to the low gas densities that imply a low accretion rate, even taking into account that the energy barrier between $\mathrm{SiO}$ molecules and silicate grain surface has been experimentally found to be zero even at low temperatures (Rouillé et al. 2015). One way of increasing the depletion of the gas elements in the ISM and the size of dust grains is by forming icy mantles around the bulk of the grain in dense molecular clouds, where the density is higher than in the diffuse medium. The icy mantle formed around dust grains can prevent the process of grain accretion by creating an energy barrier between molecules in the gas phase and the bulk of the grain. Icy mantles can however also partly evaporate when stars are formed. Such a process might be particularly efficient if the SFR is relatively high, as is the case for DGS galaxies and LBGs. Ceccarelli et al. (2018) further developed the idea proposed by Ferrara et al. (2016) by studying how 
the chemistry of the icy mantles changes as a function of different parameters, including the metallicity. The aim of such a work is to estimate the probability for Si-bearing species to encounter eachother in the icy mantle and to form clusters that can build up silicate grains. These latter authors found a very low probability for cluster formation due to this process, especially at the low metallicities that characterise the galaxies considered in the present study. Conversely, the results of a recent experiment suggest that silicate and carbon dust may be formed on the grain surface following the evaporation of icy mantles (Rouillé et al. 2020). Other laboratory experiments and theoretical works suggest that a non-negligible amount of dust can be formed in low-temperature dusty plasma (Bleecker et al. 2006; Hollenstein 2000). However, the conditions under which such a process is relevant (e.g. intensity of the radiation field providing different ionisation fractions for the elements) have only been investigated for the typical conditions of the Milky Way (Zhukovska et al. 2016).

The inability of models to reproduce the largest sMdust observed may suggest that dust condensation in SN remnants might be the preferred scenario to reproduce the observations discussed in this paper.

However, how much dust is produced and destroyed in the SN remnants is still highly debated. A large amount of dust $\left(0.1-0.5 M_{\odot}\right)$ is formed in situ in SN remnants (Matsuura et al. 2011), but it is still unclear how much dust is destroyed by the reverse shock or reformed. Matsuura et al. (2019) interpreted the increase in the $31.5 \mu \mathrm{m}$ photometry in SN1987A obtained from SOFIA FORCAST 11.1, 19.7 in 2016 with respect to 10 years earlier as a possible indication of dust re-condensation in the forward shock. Indeed, the mass of dust needed to explain the emission of this dust component at a temperature of $\sim 85 \mathrm{~K}$ is more than ten times larger than that estimated 10 years earlier. This might indicate that dust could be reformed after being destroyed by shocks. Such an interpretation should be confirmed by future observations. Indeed, another possible explanation for the observed emission is that the decrease in the density of the expanding ejecta has allowed X-rays to efficiently heat the freshly formed dust. The re-formation of dust in the post-shocked regions has also received theoretical confirmation (Sarangi et al. 2018). From an observational point of view, Gall et al. (2014) found that large dust grains are rapidly formed in SN remnants. Such grains are large enough to be able to survive the reverse shock. From the theoretical side, different authors have studied the formation and survival of dust in SN ejecta. On one hand, Bianchi \& Schneider (2007), Bocchio et al. (2016) estimated that only between $2 \%$ and $20 \%$ of the dust mass observed in SN remnants will survive the reverse shock, and that the mass of dust observed around SN1987A has not yet been destroyed by the passage of the reverse shock. On the other hand, different calculations suggest that a larger fraction (42-98\%) of the newly produced dust can survive the reverse shock in clumpy ejecta (Biscaro \& Cherchneff 2016). The survival of dust grains can also depend on their size, on the energy of the explosion, on the gas density of the ISM, and on the thickness of the hydrogen envelope (Nozawa et al. 2007).

In our work we also show that another condition to reproduce the metallicity trend is related to the initial gas content in the galaxies, which has to be at least ten times the final mass of the stars. In this context, galactic outflows are needed to efficiently remove gas and dust from the galaxy. Such a result is observationally supported for LBGs in which outflows are commonly observed (Shapley et al. 2003; Pettini et al. 2002; Gallerani et al. 2018), while star formation is rather inefficient. As far as DGS galaxies are concerned, circumgalactic dust has been detected in different galaxies included in our sample (e.g. NGC 1569, NGC 5253, He 2-10 McCormick et al. 2018; Suzuki et al. 2018). From a theoretical point of view, inflows and outflows are needed to explain the metallicity and gas content of galaxies at a redshift of 3.4 (Troncoso et al. 2014).

\section{Conclusions}

In this paper we develop and revise the existing prescriptions for dust evolution in galaxies in order to interpret the observations of DGS galaxies in the local Universe and of LBGs at high redshift.

Different investigations in the literature show the inadequacy of the current models for reproducing the largest values of the sMdust of mass observed at the largest sSFR for DGS galaxies. The inability of the current models to reproduce the observations is even more severe in our investigation, in which the sMdust and sSFR are even larger than the previous estimates in the literature (Rémy-Ruyer et al. 2015). This indicates that the state-of-theart framework adopted for interpreting the dust content in these galaxies needs to be revised. In particular, the dust prescriptions that assume low condensation efficiency for SN and dust growth in the ISM are not able to reproduce the largest sMdust at early epochs estimated for DGS galaxies and LBGs. For LBGs we develop a new description for metal and dust evolution that is in part based on the results obtained for DGS galaxies that are their nearby counterparts.

For both samples of galaxies, we reproduce the observations by adopting a top-heavy IMF which favours the fast enrichment of the ISM through SNe II and a dust condensation fraction $>25 \%$ for DGS galaxies and $>40 \%$ for LBGs. In this context, the dust enrichment from TP-AGB stars and from Type Ia SNe plays a minor role, since a top-heavy IMF is not favourable for the formation of massive stars. Galactic outflows are essential to reproduce the decline of the sMdust (and of the gas fraction for DGS galaxies) as a function of sSFR. Dust destruction by SN usually plays a secondary role with respect to dust removal from the galactic outflow. A typical star formation efficiency (the mass of gas converted into stars) of a few percent is instead required to reproduce the trends with the metallicity.

Despite the fact that grain growth is often considered to be a fundamental process to explain the amount of dust observed, we find that its contribution only starts to be relevant when the outflow begins to remove dust from the ISM, and therefore its presence would be masked by the efficient removal of dust from the galaxies.

Acknowledgements. AN acknowledges the support of the Centre National d'Etudes Spatiale (CNES) through a post-doctoral fellowship. PT work was supported by the Programme National "Physique et Chimie du Milieu Interstellaire" (PCMI) of CNRS/INSU with INC/INP co-funded by CEA and CNES. BC was supported by the ERC Consolidator Grant (Hungary) funding scheme (Project RADIOSTAR, G. A. n. 724560). HH thanks the Ministry of Science and Technology for support through grant MOST 107-2923-M-001-003-MY3 and MOST 108-2112-M-001-007-MY3, and the Academia Sinica for Investigator Award AS-IA-109-M02. We are grateful to the anonymous referee for carefully reading the manuscript and for helping in improving it.

\section{References}

Agúndez, M., \& Wakelam, V. 2013, Chem. Rev., 113, 8710

Aoyama, S., Hou, K.-C., Shimizu, I., et al. 2017, MNRAS, 466, 105

Asano, R. S., Takeuchi, T. T., Hirashita, H., \& Inoue, A. K. 2013, EPS, 65, 213

Bianchi, S., \& Schneider, R. 2007, MNRAS, 378, 973

Biscaro, C., \& Cherchneff, I. 2016, A\&A, 589, A132 
Bleecker, K., Bogaerts, A., \& Goedheer, W. 2006, Phys. Rev. E, Stat. Nonlinear Soft Matter Phys., 73, 026405

Bocchio, M., Marassi, S., Schneider, R., et al. 2016, A\&A, 587, A157

Boquien, M., Burgarella, D., Roehlly, Y., et al. 2019, A\&A, 622, A103

Bruzual, G., \& Charlot, S. 2003, MNRAS, 344, 1000

Burgarella, D., Nanni, A., Hirashita, H., et al. 2020, A\&A, 637, A32

Cai, Z.-Y., Zotti, G. D., \& Bonato, M. 2020, ApJ, 891, 74

Calura, F., Pipino, A., \& Matteucci, F. 2008, A\&A, 479, 669

Calura, F., Gilli, R., Vignali, C., et al. 2014, MNRAS, 438, 2765

Ceccarelli, C., Viti, S., Balucani, N., \& Taquet, V. 2018, MNRAS, 476, 1371

Chabrier, G. 2003, PASP, 115, 763

Côté, B., O'Shea, W., Ritter, C., Herwig, F., \& Venn, K. A. 2017, ApJ, 835, 128

Cristallo, S., Straniero, O., Piersanti, L., \& Gobrecht, D. 2015, ApJS, 219, 40

Dabringhausen, J., Kroupa, P., Pflamm-Altenburg, J., \& Mieske, S. 2012, ApJ, 747,72

da Cunha, E., Eminian, C., Charlot, S., \& Blaizot, J. 2010, MNRAS, 403, 1894 De Vis, P., Gomez, H. L., Schofield, S. P., et al. 2017, MNRAS, 471, 1743

De Vis, P., Jones, A., Viaene, S., et al. 2019, A\&A, 623, A5

Dwek, E. 1998, ApJ, 501, 643

Dwek, E., Galliano, F., \& Jones, A. P. 2007, ApJ, 662, 927

Ferrara, A., Viti, S., \& Ceccarelli, C. 2016, MNRAS, 463, L112

Gail, H. P., \& Sedlmayr, E. 1999, A\&A, 347, 594

Gall, C., Andersen, A. C., \& Hjorth, J. 2011a, A\&A, 528, A13

Gall, C., Andersen, A. C., \& Hjorth, J. 2011b, A\&A, 528, A14

Gall, C., Hjorth, J., Watson, D., et al. 2014, Nature, 511, 326

Gallerani, S., Pallottini, A., Feruglio, C., et al. 2018, MNRAS, 473, 1909

Geha, M., Brown, T. M., Tumlinson, J., et al. 2013, ApJ, 771, 29

Ginolfi, M., Graziani, L., Schneider, R., et al. 2018, MNRAS, 473, 4538

Gioannini, L., Matteucci, F., \& Calura, F. 2017, MNRAS, 471, 4615

Gomez, H. L., Clark, C. J. R., Nozawa, T., et al. 2012, MNRAS, 420, 3557

Graziani, L., Schneider, R., Ginolfi, M., et al. 2020, MNRAS, 494, 1071

Heger, A., \& Woosley, S. E. 2010, ApJ, 724, 341

Herwig, F. 2005, ARA\&A, 43, 435

Hirashita, H. 1999, ApJ, 522, 220

Hirashita, H. 2000, PASJ, 52, 585

Hirashita, H. 2012, MNRAS, 422, 1263

Hirashita, H., \& Kuo, T.-M. 2011, MNRAS, 416, 1340

Höfner, S., \& Olofsson, H. 2018, A\&ARv, 26, 1

Hollenstein, C. 2000, Plasma Phys. Controlled Fusion, 42, R93

Iwamoto, K., Brachwitz, F., Nomoto, K., et al. 1999, ApJS, 125, 439

Karakas, A. I. 2010, MNRAS, 403, 1413

Kobayashi, C., Umeda, H., Nomoto, K., Tominaga, N., \& Ohkubo, T. 2006, ApJ, 653,1145

Leśniewska, A., \& Michałowski, M. J. 2019, A\&A, 624, L13

Limongi, M., \& Chieffi, A. 2018, ApJS, 237, 13

Lisenfeld, U., \& Ferrara, A. 1998, ApJ, 496, 145

Liu, H.-M., \& Hirashita, H. 2019, MNRAS, 490, 540

Lodders, K. 2010, Astrophys. Space Sci. Proc., 16, 379

Madden, S. C., Rémy-Ruyer, A., Galametz, M., et al. 2013, PASP, 125, 600

Mancini, M., Schneider, R., Graziani, L., et al. 2015, MNRAS, 451, L70
Marassi, S., Schneider, R., Limongi, M., et al. 2019, MNRAS, 484, 2587 Marks, M., Kroupa, P., Dabringhausen, J., \& Pawlowski, M. S. 2012, MNRAS, 422, 2246

Mathis, J. S., Rumpl, W., \& Nordsieck, K. H. 1977, ApJ, 217, 425

Matsuura, M., Dwek, E., Meixner, M., et al. 2011, Science, 333, 1258

Matsuura, M., De Buizer, J. M., Arendt, R. G., et al. 2019, MNRAS, 482, 1715

Mattsson, L. 2020, MNRAS, 491, 4334

Mattsson, L., Fynbo, J. P. U., \& Villarroel, B. 2019, MNRAS, 490, 5788

McCormick, A., Veilleux, S., Meléndez, M., et al. 2018, MNRAS, 477, 699

McKee, C. 1989, in Interstellar Dust, eds. L. J. Allamandola, \& A. G. G. M. Tielens , IAU Symp., 135, 431

McKinnon, R., Vogelsberger, M., Torrey, P., Marinacci, F., \& Kannan, R. 2018, MNRAS, 478, 285

McWilliam, A., Wallerstein, G., \& Mottini, M. 2013, ApJ, 778, 149

Michałowski, M. J. 2015, A\&A, 577, A80

Murray, N., Quataert, E., \& Thompson, T. A. 2005, ApJ, 618, 569

Nanni, A., Bressan, A., Marigo, P., \& Girardi, L. 2013, MNRAS, 434, 2390

Nanni, A., Bressan, A., Marigo, P., \& Girardi, L. 2014, MNRAS, 438, 2328

Nomoto, K., Kobayashi, C., \& Tominaga, N. 2013, ARA\&A, 51, 457

Nozawa, T., Kozasa, T., Umeda, H., Maeda, K., \& Nomoto, K. 2003, ApJ, 598, 785

Nozawa, T., Kozasa, T., \& Habe, A. 2006, ApJ, 648, 435

Nozawa, T., Kozasa, T., Habe, A., et al. 2007, ApJ, 666, 955

Nozawa, T., Maeda, K., Kozasa, T., et al. 2011, ApJ, 736, 45

Pettini, M., Rix, S. A., Steidel, C. C., et al. 2002, Ap\&SS, 281, 461

Pforr, J., Maraston, C., \& Tonini, C. 2012, MNRAS, 422, 3285

Popping, G., Somerville, R. S., \& Galametz, M. 2017, MNRAS, 471, 3152

Rémy-Ruyer, A., Madden, S. C., Galliano, F., et al. 2013, A\&A, 557, A95

Rémy-Ruyer, A., Madden, S. C., Galliano, F., et al. 2014, A\&A, 563, A31

Rémy-Ruyer, A., Madden, S. C., Galliano, F., et al. 2015, A\&A, 582, A121

Ritter, C., Herwig, F., Jones, S., et al. 2018, MNRAS, 480, 538

Rouillé, G., Jäger, C., Krasnokutski, S. A., Krebsz, M., \& Henning, T. 2015, Faraday Discuss., 168, 449

Rouillé, G., Jäger, C., \& Henning, T. 2020, ApJ, 892, 96

Rowlands, K., Gomez, H. L., Dunne, L., et al. 2014, MNRAS, 441, 1040

Sarangi, A., Dwek, E., \& Arendt, R. G. 2018, ApJ, 859, 66

Schneider, R., Hunt, L., \& Valiante, R. 2016, MNRAS, 457, 1842

Shapley, A. E., Steidel, C. C., Pettini, M., \& Adelberger, K. L. 2003, ApJ, 588, 65

Spyromilio, J., Leibundgut, B., \& Gilmozzi, R. 2001, A\&A, 376, 188

Suzuki, T., Kaneda, H., Onaka, T., et al. 2018, MNRAS, 477, 3065

Troncoso, P., Maiolino, R., Sommariva, V., et al. 2014, A\&A, 563, A58

Valiante, R., Schneider, R., Salvadori, S., \& Gallerani, S. 2014, MNRAS, 444, 2442

Ventura, P., Criscienzo, M. D., Schneider, R., et al. 2012, MNRAS, 424, 2345

Wang, W.-C., Hirashita, H., \& Hou, K.-C. 2017, MNRAS, 465, 3475

Ysard, N., Koehler, M., Jimenez-Serra, I., Jones, A. P., \& Verstraete, L. 2019, A\&A, 631, A88

Zhukovska, S. 2014, A\&A, 562, A76

Zhukovska, S., Dobbs, C., Jenkins, E. B., \& Klessen, R. S. 2016, ApJ, 831, 147 


\section{Appendix A: Properties of DGS galaxies and best-fitting models from cIGALE}

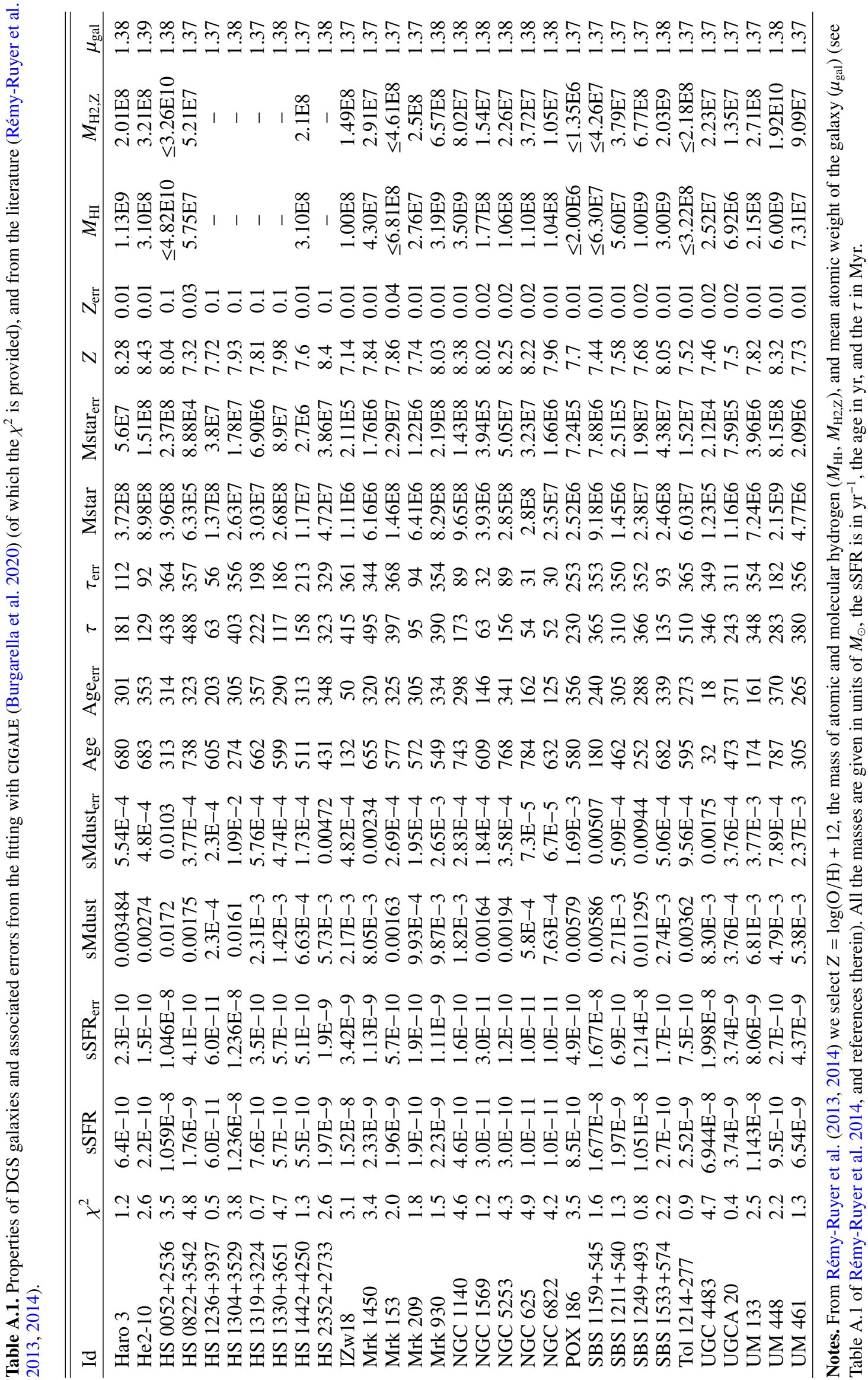


A. Nanni et al.: The gas, metal, and dust evolution in low-metallicity local and high-redshift galaxies
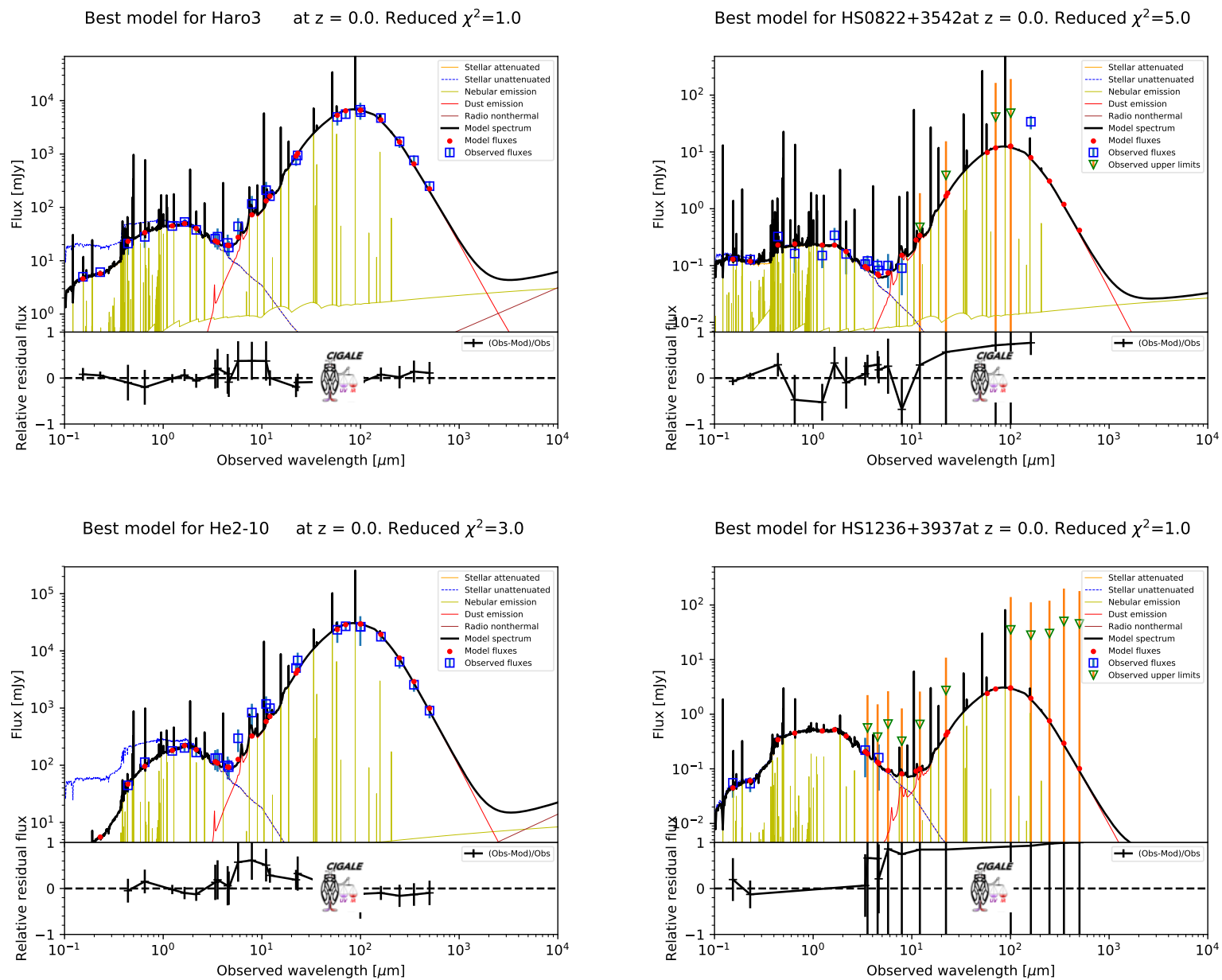

Best model for HS0052 +2536 at $z=0.0$. Reduced $\chi^{2}=4.0$

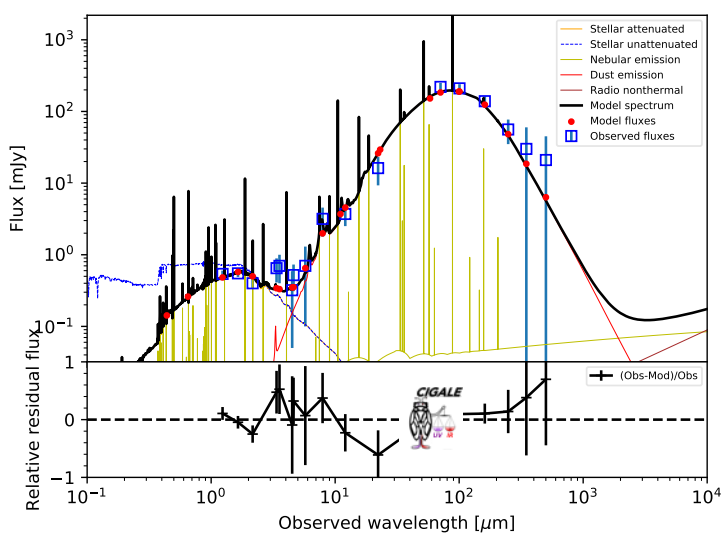

Best model for HS1304+3529at $z=0.0$. Reduced $\chi^{2}=4.0$

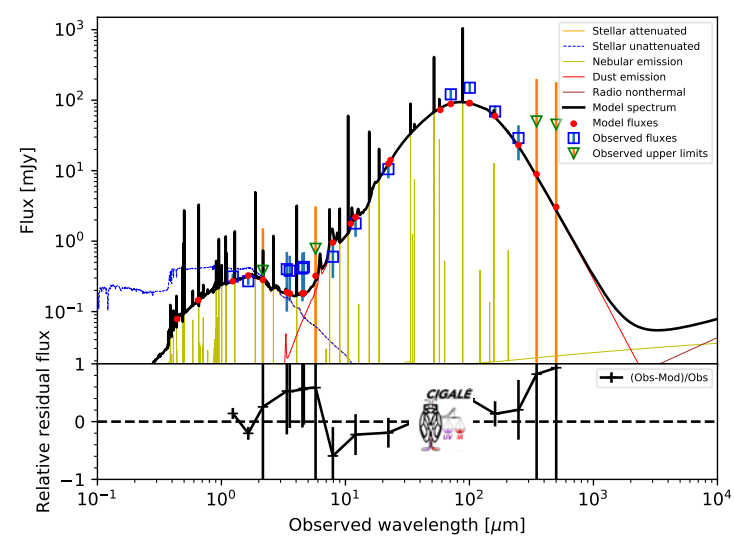

Fig. A.1. Modelled SEDs overplotted on the data for the samples of galaxies used in this paper. Note that the modelled SED shown here are the best models for each object. They might not capture the entire information derived from the Bayesian analysis. 

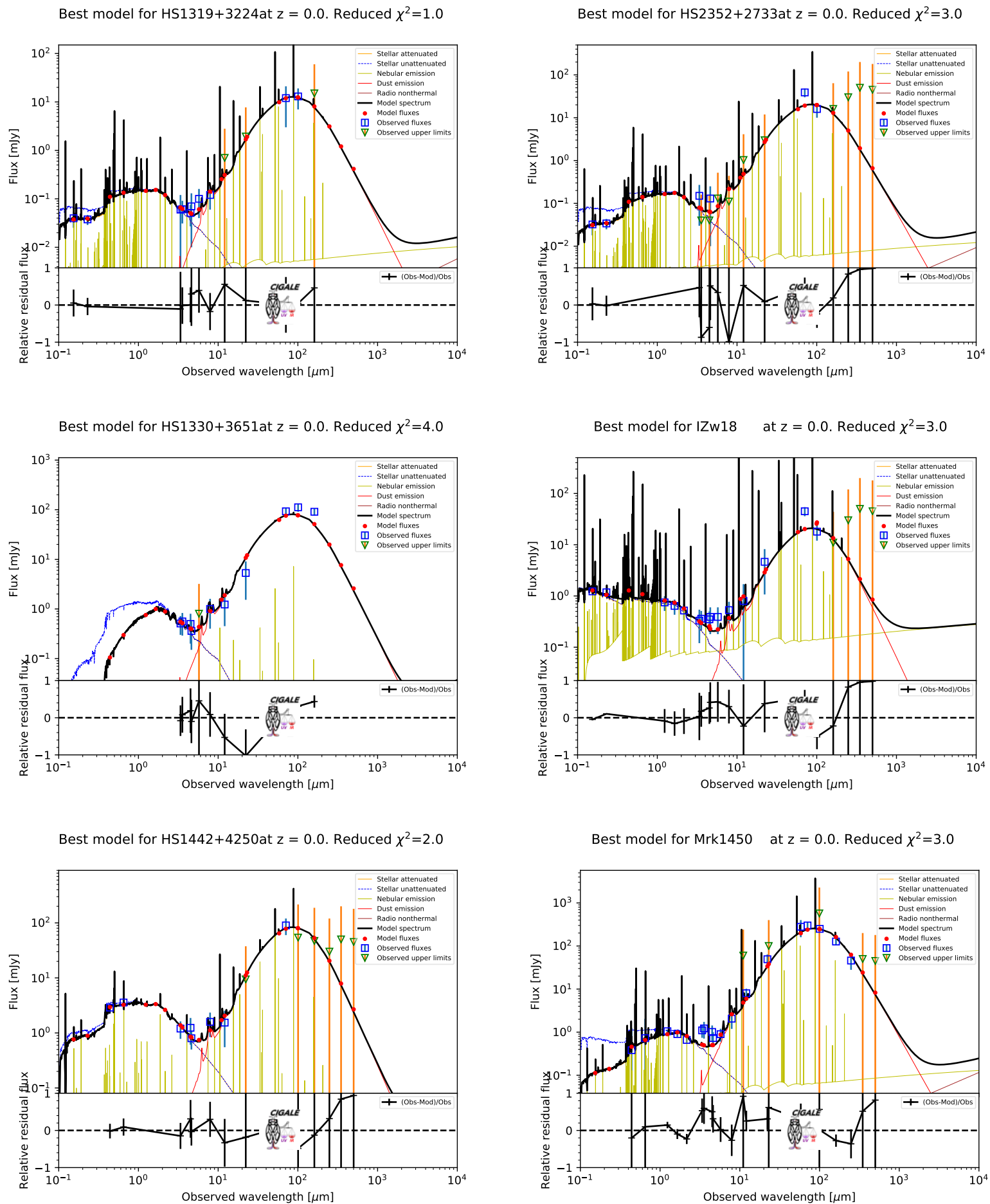

Fig. A.1. continued. 
A. Nanni et al.: The gas, metal, and dust evolution in low-metallicity local and high-redshift galaxies
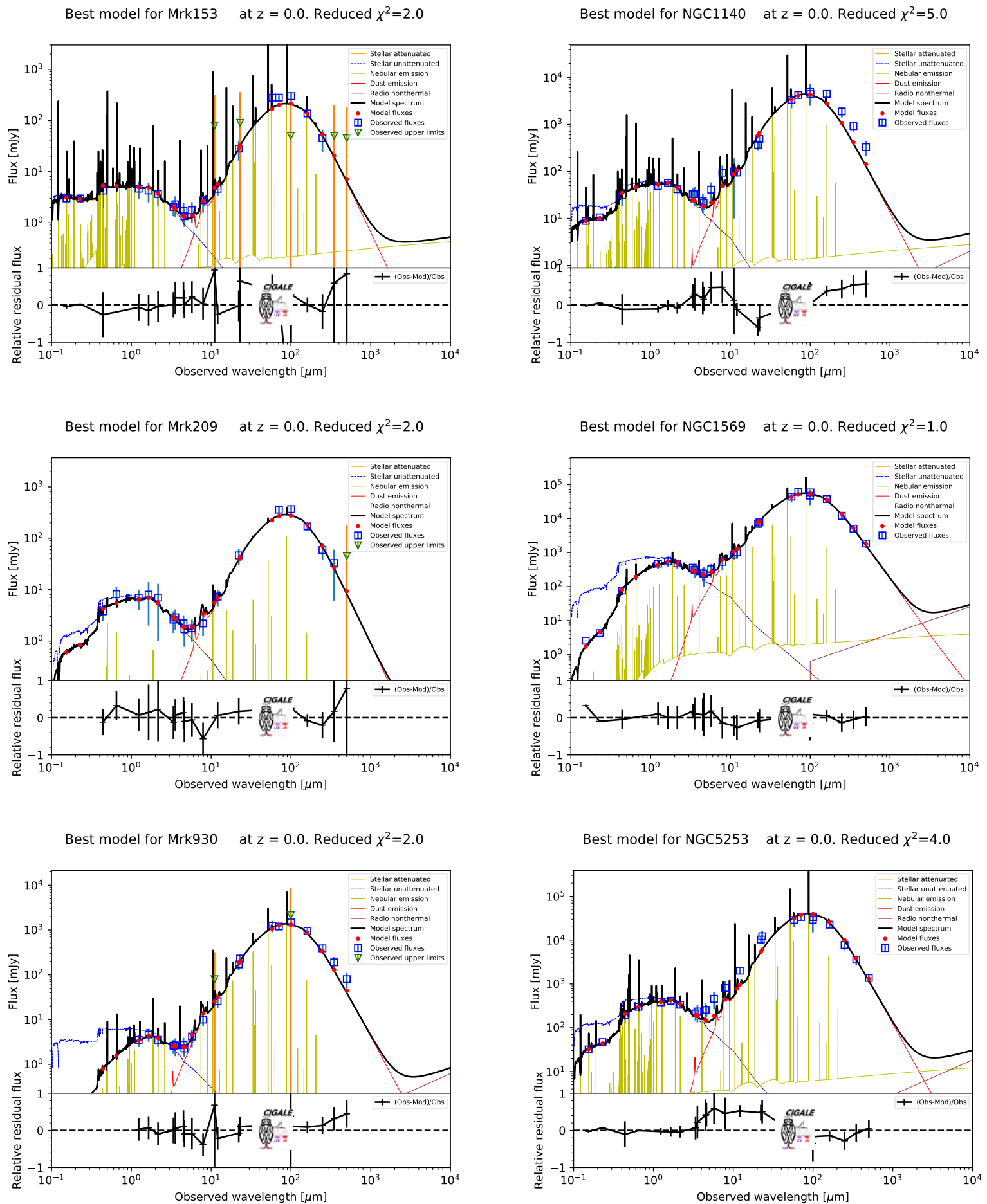

Fig. A.1. continued. 

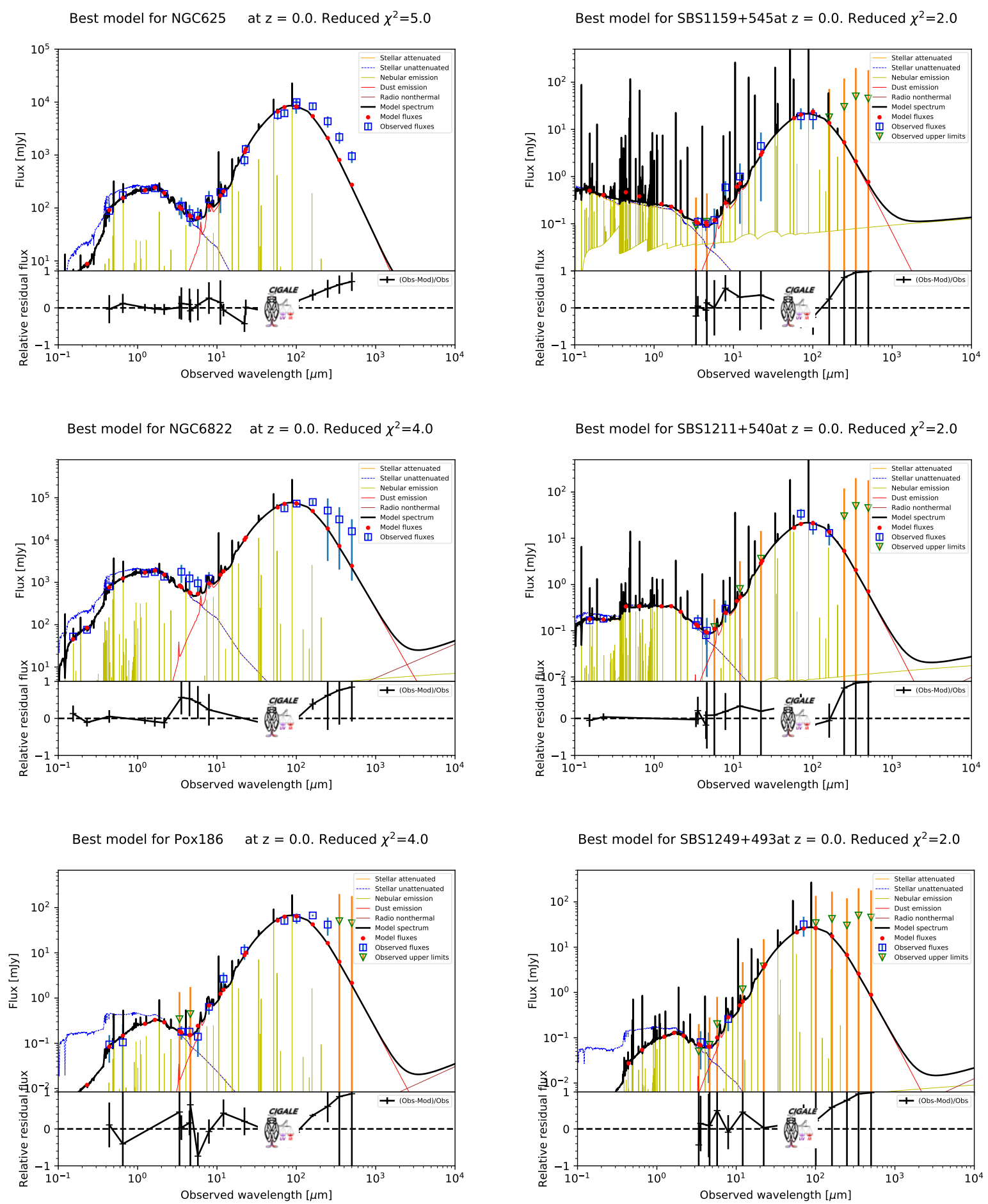

Fig. A.1. continued. 
A. Nanni et al.: The gas, metal, and dust evolution in low-metallicity local and high-redshift galaxies
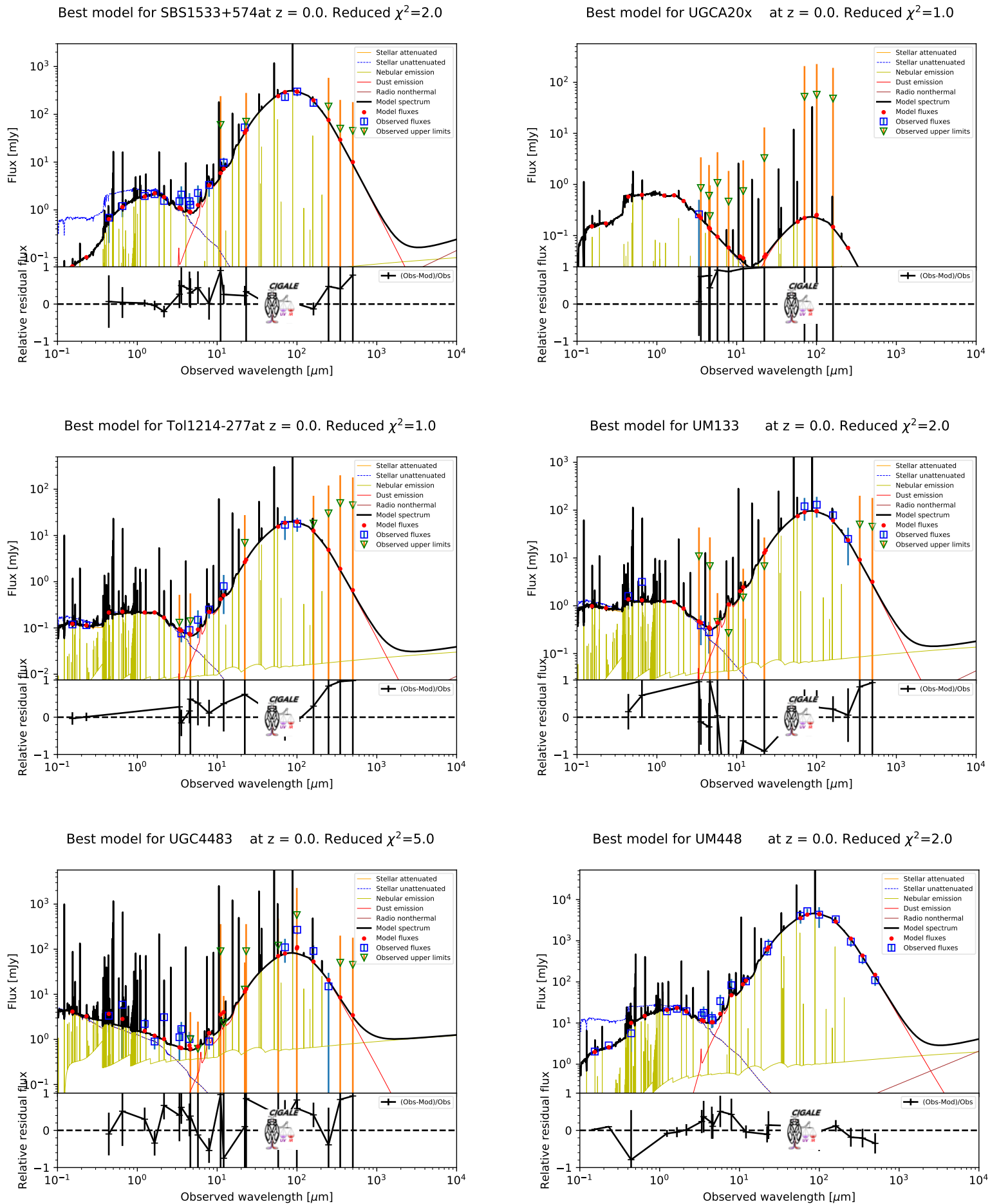

Fig. A.1. continued. 
A\&A 641, A168 (2020)

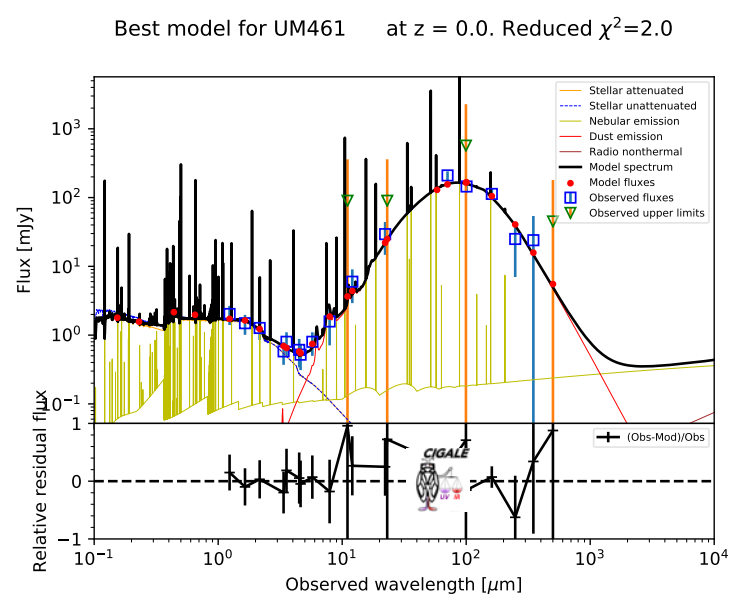

Fig. A.1. continued. 